\title{
Zeros of amplitude in the associated production of photon and leptoquark at $e-p$ collider
}

\author{
Priyotosh Bandyopadhyay $^{\mathrm{a}}\left(\mathbb{D}\right.$, Saunak Dutta $^{\mathrm{b}} \mathbb{D}$, Anirban Karan $^{\mathrm{c}}{ }_{\mathbb{C}}$ \\ Indian Institute of Technology Hyderabad, Kandi, Sangareddy, Telangana 502285, India
}

Received: 4 January 2021 / Accepted: 28 March 2021 / Published online: 13 April 2021

(C) The Author(s) 2021

\begin{abstract}
Though various extensions of the Standard Model with higher gauge group predict the existence of leptoquarks, none of them has been observed yet at any of the colliders. In this paper, we study the prospect of several past and future $e-p$ colliders like HERA, LHeC and FCC-he to detect them through radiation amplitude zero. We find that the leptoquarks showing zeros in the tree-level single-photon amplitudes at $e-p$ collider lie within the complementary set of those exhibiting zeros at $e-\gamma$ collider. We present a PYTHIA-based analysis for HERA, LHeC and FCC-he (run II) to detect the leptoquarks with masses $70 \mathrm{GeV}, 900 \mathrm{GeV}$ and $1.5 \mathrm{TeV}(2.0$ $\mathrm{TeV}$ ) respectively through radiation amplitude zero.
\end{abstract}

\section{Contents}

1 Introduction $\ldots \ldots \ldots \ldots \ldots \ldots$

2 Theoretical Framework . . . . . . . . . . . . . 2

3 Experimental bounds and benchmark points . . . . . 4

4 Set up for the collider simulation . . . . . . . . 7

5 The hadron-electron ring accelerator (HERA) . . . . 8

5.1 Scalar Doublet . . . . . . . . . . . . . . . 9

5.2 Scalar Singlet . . . . . . . . . . . . 11

5.3 Scalar Triplet . . . . . . . . . . . . . . 12

5.4 Vector Singlet . . . . . . . . . . . . . . . . . 14

5.5 Vector Doublet . . . . . . . . . . . . . . 15

5.6 Vector Triplet . . . . . . . . . . . . . . 15

6 Large hadron electron collider $(\mathrm{LHeC})$. . . . . . 17

7 FCC-he . . . . . . . . . . . . . . 18

7.1 FCC I . . . . . . . . . . . . 18

7.2 FCC-II . . . . . . . . . . . . . . 21

8 Discussion and conclusion .......... . 21

References ................. 23

a e-mail: bpriyo@phy.iith.ac.in

be-mail: ph17resch11002@iith.ac.in

c e-mail: kanirban@iith.ac.in (corresponding author)

\section{Introduction}

The phenomenon of radiation amplitude zero (RAZ) was discovered long ago by Brown, Mikaelian, Sahdev, and Samuel $[1,2]$ in the context of probing electromagnetic properties of $W$-boson. Soon after, it was noticed that in case of any gauge theory tree-level amplitude for any four-particle process involving one or more massless gauge fields in external legs gets factorized into two parts comprehending several internal symmetry (charge) dependence and spin (polarization) dependence separately governed by a spatial generalized Jacobi identity [3,4]. Hinging on charge and four momenta of the four external particles, the first factor for single photon ${ }^{1}$ tree-level amplitude vanishes sometimes at certain regions of phase space and thus RAZ occurs. Later, RAZ was realized as a relativistic and quantum mechanical generalization of the classical event displaying no dipole radiation in the collision of non-relativistic particles with same charge-to-mass ratio and $g$-factor $[6,7]$. Thus, the kinematic condition for single photon amplitude at tree-level to vanish is that the ratio $\left(Q_{i} / p_{i} \cdot k\right)$ must be same for all external particles (labelled by $i$ ) where their charges and four momenta are given by $Q_{i}$ and $p_{i}^{\mu}$ respectively with $k^{\mu}$ being the four momentum of the photon $[6,7]$. The criteria for these zeros to lie inside the kinematically allowed region of phase space are also well-studied [8-10]. Although various contaminations like initial and final state photon radiations, QED and QCD loop contributions, uncertainties in partonic centre of momentum reconstruction, etc., smear the effect of RAZ [1119], noticeable dip in the angular distribution still remains [20-22].

On the other hand, leptoquarks are electromagnetically charged, colour triplet proposed bosons that emerge naturally in several extensions of the Standard Model (SM) uni-

\footnotetext{
1 The tree-level factorization holds for single gluon amplitude also, but the angular distribution does not show any zero due to colour summation $[5]$.
} 
fying matter fields [23-32]. The peculiarity of these particles is that they carry both non-zero lepton and baryon numbers simultaneously and therefore are able to transform a quark into a lepton or vice-versa [33,34]. Much effort has been devoted through last few decades for the direct detection of these particles at different colliders [35-88], but no conclusive evidence advocating their existence has been discovered so far. In this paper, we have scrutinized the possibility of detecting all kinds of leptoquarks (if there is any), that can be produced at any electron-proton collider, through the phenomenon of RAZ. Though hadronic colliders have the splendour of performing collisions at very high energy, the main disadvantage there is the emergence of large SM background which makes the searches for beyond Standard Model (BSM) scenarios unfathomable. In contrast, electronhadron and electron-photon colliders produce extremely limited number of SM processes as background making them preferable for any BSM hunt. The drawback of lower centre of momentum energy for these kind of machines will be tackled in various proposed colliders that are going to be built in near future with $\mathrm{TeV}$ scale energy.

Based on the centre of momentum (CM) energies for various past and future $e-p$ colliders [89-92] like HERA, LHeC and FCC-he (run II), we analyse the production channels of leptoquarks for masses $70 \mathrm{GeV}, 900 \mathrm{GeV}$ and $1.5 \mathrm{TeV}(2.0$ $\mathrm{TeV}$ ) respectively associated with a photon. Though current experimental bounds seem to rule out leptoquarks with mass below $1.5 \mathrm{TeV}$, most of those analyses are performed surmising the coupling of leptoquarks to one generation of quark and lepton only. While considering their coupling to all the generations, the constraints become more relaxed and it turns out that the above mentioned masses are still allowed depending on specific couplings to different quarks and leptons. Now, if a leptoquark is produced, it will eventually decay to a quark and a lepton; therefore we look for mono-jet plus monolepton events associated with single photon at the detector for our analysis. In order to keep SM background null, we leave the electron events and search for the muon events only. In a PYTHIA-based simulation, we reconstruct the leptoquark from the invariant mass of the muon-jet pair, then we boost the whole system back to the CM frame and finally study the angular distribution of the process with respect to the angle between radiated photon and electron beam. Using the charge separation of final state jets, model backgrounds have also been estimated. Unlike $e-\gamma$ collider [78-80,86], the zeros of single-photon tree-level amplitude in this case do not depend on masses of leptoquarks or energies of collisions, rather they are controlled solely by electromagnetic charges of leptoquarks. However, it is very important to notice that the leptoquarks showing RAZ at $e-p$ collider lie in the complementary set of those displaying the zero in single-photon tree-level amplitude at $e-\gamma$ collider. It is also noteworthy that while distinguishing signatures of different leptoquarks, $e-\gamma$

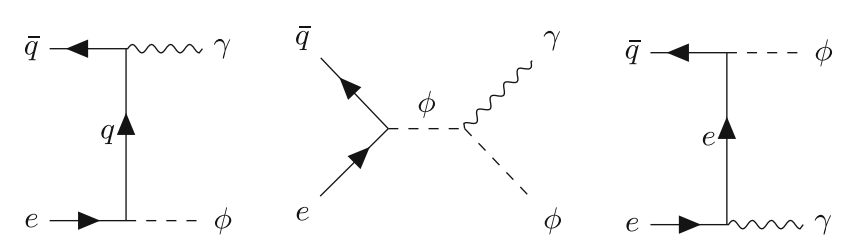

Fig. 1 Feynman diagrams for $e \bar{q} \rightarrow \gamma \phi$

and $e-p$ colliders have great advantage over $p p$ or $p \bar{p}$ colliders. Although the angular distribution of pair production in hadronic colliders can categorize the leptoquarks according to their spins, it fails to separate various $S U(2)_{L}$ multiplets with same spin. In this context, $e-p$ and $e-\gamma$ colliders together do this job through zeros of single photon tree level amplitude.

This paper is organized in the following way. The forthcoming section (Sect. 2) illustrates the theoretical description of RAZ for various scalar and vector leptoquarks. Bounds on masses, couplings and branching fractions of leptoquarks from different experiments and specification of benchmark points for our simulation have been summarized in Sect. 3 . The succeeding section (Sect. 4) deals with the set up needed for this collider simulation. In Sect. 5, we reconsider the aspects of HERA for the search of low mass leptoquarks. The prospects of $\mathrm{LHeC}$ and FCC-he in detecting the heavy leptoquarks have been demonstrated in the next two sections (Sects. 6, 7). We finally sum up and conclude in Sect. 8.

\section{Theoretical Framework}

In this section, we discuss the theoretical aspects of observing RAZ at $e-p$ collider for the production of any leptoquark $\phi$ associated with a photon. The parton level interactions responsible for this process are $e \bar{q}$ (or $q) \rightarrow \gamma \phi$. We present here the analytic calculations for $e \bar{q} \rightarrow \gamma \phi$ only. It is straight forward to find the expressions for the other parton level process by repeating the same procedure.

There are three Feynman diagrams contributing to the process $e \bar{q} \rightarrow \gamma \phi$ as shown in Fig. 1. Combining the matrix elements for all the diagrams, the total amplitudes for production of scalar and vector leptoquarks along with a photon from the collision of electrons and anti-quarks are respectively given by:

$$
\begin{aligned}
\mathcal{M}^{S}= & -i e\left(\frac{Q_{\phi}}{p_{\phi} \cdot k}+\frac{1}{p_{e} \cdot k}\right) \epsilon_{\mu}^{\gamma *} \bar{v}\left(p_{\bar{q}}\right)\left(Y_{L}^{e q} P_{L}+Y_{R}^{e q} P_{R}\right) \\
& \times\left[p_{\phi}^{\mu}-\left(\frac{p_{\phi} \cdot k}{p_{\bar{q}} \cdot k}\right)\left(p_{\bar{q}}^{\mu}-\frac{1}{2} \gamma^{\mu} \not k\right)\right] u\left(p_{e}\right), \\
\mathcal{M}^{V}= & -i e\left(\frac{Q_{\phi}}{p_{\phi} \cdot k}+\frac{1}{p_{e} \cdot k}\right) \epsilon_{\mu}^{\gamma *} \epsilon_{v}^{\phi *} \bar{v}\left(p_{\bar{q}}\right)\left[\frac{1}{2} \gamma^{v} \gamma^{\mu} / k\right.
\end{aligned}
$$




$$
\begin{aligned}
& \left.+\gamma^{v} p_{e}^{\mu}+\left(\frac{p_{e} \cdot k}{p_{\bar{q}} \cdot k}\right)\left(\gamma^{\mu} p_{e}^{v}+\frac{1}{2} \gamma^{\mu} \gamma^{v} p_{\phi}\right)\right] \\
& \times\left(Y_{L}^{e q} P_{L}+Y_{R}^{e q} P_{R}\right) u\left(p_{e}\right),
\end{aligned}
$$

where $p_{e}^{\mu}, p_{\bar{q}}^{\mu}, p_{\phi}^{\mu}$, and $k^{\mu}$ are the four momenta of electron, anti-quark, leptoquark and photon respectively, $Y_{L, R}^{e q}$ are the couplings of leptoquark with different generations of lefthanded and right-handed leptons and quarks, $-e$ and $Q_{\phi} e$ denote the charges of electron and leptoquark respectively, $\epsilon_{\mu}^{\gamma, \phi}$ signify the polarizations of the photon and the vector leptoquark and $P_{L, R} \equiv\left(1 \mp \gamma^{5}\right) / 2$. While deriving amplitudes in Eqs. (1) and (2), we have presumed the electron and anti-quark to be massless. However, at the time of simulation, we have taken the masses of the SM fermions accordingly.

The factorization of the single-photon tree-level amplitude for the above-mentioned four-particle process, as described in Refs. [3-5], is quite apparent from Eqs. (1) and (2). The charge-dependent factor $\left(\frac{Q_{\phi}}{p_{\phi} \cdot k}+\frac{1}{p_{e} \cdot k}\right)$ in these two equations vanishes at particular points of phase space for some leptoquarks $[6,7]$ which in turn causes RAZ. Therefore, if $\theta^{*}$ be the angle between photon and electron in $\mathrm{CM}$ frame at which RAZ happens, then:

$\cos \theta^{*}=1+\frac{2}{Q_{\phi}}$

This implies that the necessary condition for RAZ occurring inside the physical region of an $e-p$ collider is given by:

$Q_{\phi}<-1$.

In Table 1, we epitomize all the leptoquarks that can be produced at $e-p$ collider and indicate which of them will show RAZ. Refs. [33,34,61-63,93] contain comprehensive list of all the leptoquarks and we follow similar notations. Reference [94] also performed similar study with generic exotic scalars. The subscript 1,2 and 3 in the name of leptoquarks signify singlet, doublet and triplet leptoquarks under $S U(2)_{L}$ gauge group; additionally, the presence of Lorentz index $\mu$ indicates the corresponding leptoquark to be a vector particle. In Table 1, we have also mentioned the weak hypercharge $\left(\mathrm{Y}_{\phi}\right)$, the third component of weak isospin $\left(T_{3}\right)$ and electromagnetic charge $\left(Q_{\phi}\right)$ of different leptoquarks. The gauge representations of all the leptoquark multiplets have been explicitly mentioned in the subsequent subsections of Sect. 5 where they are introduced and discussed. However, while discussing a particular component of a doublet or triplet leptoquark, we explicitly mention its electromagnetic charge in the superscript to distinguish it from the other excitations of the same multiplet.

It is noteworthy from Table 1 that $S_{3}^{-2 / 3}, \widetilde{R}_{2}^{-1 / 3}, \widetilde{V}_{2 \mu}^{-2 / 3}$ and $U_{3 \mu}^{-1 / 3}$ will never be produced at $e$ - $p$ collider since the gaugestructure of the Lagrangian prohibits them to interact with
Table 1 Specification of different leptoquarks produced at $e-p$ collider. We follow the notations introduced in Refs. [33,34,61-63,93] where a comprehensive studies of all leptoquark models have been carried out

\begin{tabular}{llllll}
\hline$\phi$ & $\mathrm{Y}_{\phi}$ & $T_{3}$ & $Q_{\phi}$ & Production channel & $\cos \theta^{*}$
\end{tabular}

Scalar leptoquarks

$\begin{array}{lcccll}S_{1} & 2 / 3 & 0 & 1 / 3 & e^{-} u \rightarrow \gamma\left(S_{1}^{+1 / 3}\right)^{c} & - \\ \widetilde{S}_{1} & 8 / 3 & 0 & 4 / 3 & e^{-} d \rightarrow \gamma\left(\widetilde{S}_{1}^{+4 / 3}\right)^{c} & -1 / 2 \\ R_{2} & 7 / 3 & 1 / 2 & 5 / 3 & e^{-} \bar{u} \rightarrow \gamma\left(R_{2}^{+5 / 3}\right)^{c} & -1 / 5 \\ & & -1 / 2 & 2 / 3 & e^{-} \bar{d} \rightarrow \gamma\left(R_{2}^{+2 / 3}\right)^{c} & - \\ \widetilde{R}_{2} & 1 / 3 & 1 / 2 & 2 / 3 & e^{-} \bar{d} \rightarrow \gamma\left(\widetilde{R}_{2}^{+2 / 3}\right)^{c} & - \\ & & -1 / 2 & -1 / 3 & - & - \\ S_{3} & 2 / 3 & 1 & 4 / 3 & e^{-} d \rightarrow \gamma\left(S_{3}^{+4 / 3}\right)^{c} & -1 / 2 \\ & & 0 & 1 / 3 & e^{-} u \rightarrow \gamma\left(S_{3}^{+1 / 3}\right)^{c} & - \\ & & -1 & -2 / 3 & - & \end{array}$

\begin{tabular}{|c|c|c|c|c|c|}
\hline$U_{1 \mu}$ & $4 / 3$ & 0 & $2 / 3$ & $e^{-\bar{d}} \rightarrow \gamma\left(U_{1 \mu}^{+2 / 3}\right)^{c}$ & - \\
\hline$\widetilde{U}_{1 \mu}$ & $10 / 3$ & 0 & $5 / 3$ & $e^{-} \bar{u} \rightarrow \gamma\left(\tilde{U}_{1 \mu}^{+5 / 3}\right)^{c}$ & $-1 / 5$ \\
\hline \multirow[t]{2}{*}{$V_{2 \mu}$} & $5 / 3$ & $1 / 2$ & $4 / 3$ & $e^{-} d \rightarrow \gamma\left(V_{2 \mu}^{+4 / 3}\right)^{c}$ & $-1 / 2$ \\
\hline & & $-1 / 2$ & $1 / 3$ & $e^{-} u \rightarrow \gamma\left(V_{2 \mu}^{+1 / 3}\right)^{c}$ & - \\
\hline \multirow[t]{2}{*}{$\tilde{V}_{2 \mu}$} & $-1 / 3$ & $1 / 2$ & $1 / 3$ & $e^{-} u \rightarrow \gamma\left(\widetilde{V}_{2 \mu}^{+1 / 3}\right)^{c}$ & - \\
\hline & & $-1 / 2$ & $-2 / 3$ & - & - \\
\hline \multirow[t]{3}{*}{$\boldsymbol{U}_{3 \mu}$} & $4 / 3$ & 1 & $5 / 3$ & $e^{-} \bar{u} \rightarrow \gamma\left(U_{3 \mu}^{+5 / 3}\right)^{c}$ & $-1 / 5$ \\
\hline & & 0 & $2 / 3$ & $e^{-\bar{d}} \rightarrow \gamma\left(U_{3 \mu}^{+2 / 3}\right)^{c}$ & - \\
\hline & & -1 & $-1 / 3$ & - & - \\
\hline
\end{tabular}

Vector Leptoquarks

any charged lepton. Another important remark to make at this point is that the zeros of single-photon tree-level amplitudes for production of leptoquark associated with a quark at $e-\gamma$ collider occur only if $-1<Q_{\phi}<0$ [86] which lies beyond the parameter-space for $Q_{\phi}$ specified by Eq. (4). Hence, the leptoquark whose angular distribution for the production channel does not show any zero at $e-\gamma$ collider will definitely exhibit RAZ here.

The angular distribution in $\mathrm{CM}$ frame with respect to the angle $(\theta)$ between electron and photon for the channel $e \bar{q}_{\alpha} \rightarrow \gamma \phi_{\alpha}$ ( $\alpha$ is the colour-index) is given by:

$\frac{d \sigma}{d \cos \theta}=\frac{s-M_{\phi}^{2}}{32 \pi s^{2}}\left(\frac{1}{4} \sum_{\text {spin }}\left|\mathcal{M}^{S, V}\right|^{2}\right)$,

where, $s=\left(p_{e}+p_{\bar{q}}\right)^{2}, M_{\phi}$ represents the mass of leptoquark and $\left(\sum_{\text {spin }}\left|\mathcal{M}^{S, V}\right|^{2}\right)$ indicates the sum over polarizations of all final state particles for the absolute square of matrix 
element which can be expressed for scalar and vector leptoquark cases as:

$$
\begin{aligned}
\sum_{\text {spin }}\left|\mathcal{M}^{S}\right|^{2}= & e^{2}\left[\left(Y_{L}^{e q}\right)^{2}+\left(Y_{R}^{e q}\right)^{2}\right]\left[1+\frac{\left(s+M_{\phi}^{2}\right)^{2}}{\left(s-M_{\phi}^{2}\right)^{2}}\right] \\
& \times \operatorname{cosec}^{2} \theta\left[Q_{\phi} \cos \theta-\left(2+Q_{\phi}\right)\right]^{2}, \\
\sum_{\text {spin }}\left|\mathcal{M}^{V}\right|^{2}= & 2 e^{2}\left[\left(Y_{L}^{e q}\right)^{2}+\left(Y_{R}^{e q}\right)^{2}\right]\left[\cos ^{2} \theta+\frac{\left(s+M_{\phi}^{2}\right)^{2}}{\left(s-M_{\phi}^{2}\right)^{2}}\right] \\
& \times \operatorname{cosec}^{2} \theta\left[Q_{\phi} \cos \theta-\left(2+Q_{\phi}\right)\right]^{2} .
\end{aligned}
$$

However, one should not expect Eq. (5) to hold explicitly for associate production of a leptoquark with a photon in any $e-p$ collider since the parton distribution function of proton would modify the angular distribution accordingly. Moreover, the uncertainty in the parton distribution function may also lead to slight shift in the position of RAZ.

\section{Experimental bounds and benchmark points}

This section deals with different experimental bounds on leptoquarks and choice of benchmark points for our simulation. There are several direct and indirect bounds on leptoquarks coming from different experiments. While the indirect constraints arise from restrictions on leptoquark-induced fourfermion interactions, testable at low-energy experiments, the direct bounds emerge from the possibility of their production at different high-energy colliders. The indirect constraints $^{2}$ on leptoquarks have been studied extensively in the Refs. [93,96-99]. For instance, it can be obtained from Ref. [98] that scalar leptoquarks coupling to the first generation of left-handed quark and lepton should satisfy the bound $\left(Y / \widetilde{M}_{\phi}\right)^{2} \leq 0.07$ and the similar vector leptoquarks should obey $\left(Y / \widetilde{\widetilde{M}}_{\phi}\right)^{2} \leq 0.4$ where $\widetilde{M}_{\phi}=\left(M_{\phi} / 1 \mathrm{TeV}\right)$. However, for our present purpose we mainly focus on the collider bounds.

The search for leptoquarks at different colliders has a long history. The first ever search was performed by the CELLO [35] and JADE [36] Collaborations at the PETRA for pair production of leptoquarks through $e^{+} e^{-}$collision with the centre of momentum energy $(\equiv \sqrt{s}$ ) around $40 \mathrm{GeV}$, but no evidence was found in both the detectors, and the CELLO Collaboration excluded the leptoquark mass from 7 to 20.5 $\mathrm{GeV}$. A similar bound was also set up by the AMY Collaboration [37] when no signature of leptoquark was detected in $e^{+} e^{-}$annihilation at the TRISTAN with $50 \mathrm{GeV} \leq \sqrt{s} \leq$ $60.8 \mathrm{GeV}$. The existence of leptoquark was looked for at

\footnotetext{
${ }^{2}$ Description of all the indirect bounds on leptoquarks is presented in the "Indirect Limits for Leptoquarks" section of Ref. [95].
}

another $e^{+} e^{-}$collider, the LEP, by the ALEPH, L3, OPAL and DELPHI Collaborations. Results from the ALEPH [38] and L3 [39] Collaborations excluded the mass for each generation of leptoquark (coupling to one generation of quark and lepton only) below $44 \mathrm{GeV}$. The OPAL Collaboration [40] analysed the LEP data with integrated luminosity $\left(\mathcal{L}_{\text {int }}\right)$ of $596 \mathrm{pb}^{-1}$ and centre of momentum energy ranging from $189 \mathrm{GeV}$ to $209 \mathrm{GeV}$ to provide the lower limit $^{3}$ on $M_{\phi}$ being below $100 \mathrm{GeV}$. On the other hand, the last update from the DELPHI Collaboration [41] used the LEP-2 data with $\sqrt{s}=$ $183 \mathrm{GeV}$ and integrated luminosity of $47.4 \mathrm{pb}^{-1}$ and constrained the couplings of scalar and vector leptoquarks with varying $M_{\phi}$. According to their analysis, the lower limit on the masses of first generation scalar (vector) leptoquarks with coupling $Y=e$ and $100 \%$ branching to charged lepton mode $(\beta)$ were $161 \mathrm{GeV}(171 \mathrm{GeV})$ for $Q_{\phi}=1 / 3,5 / 3$ and 134 $\mathrm{GeV}(150 \mathrm{GeV})$ for $Q_{\phi}=2 / 3,4 / 3$ at $95 \%$ confidence level (C.L.).

A large number of investigations for the existence of both scalar and vector leptoquarks have been performed by the $\mathrm{H} 1$ and ZEUS detectors at the $e-p$ collider HERA too. In the last update from the $\mathrm{H} 1$ Collaboration [43], full data with $\mathcal{L}_{i n t}=$ $446 \mathrm{pb}^{-1}$ has been used to rule out the first generation of leptoquark with mass up to $800 \mathrm{GeV}$ at $95 \%$ C.L. for leptoquark coupling $Y=e$. Their previous study [42] was performed for full data sample at $\sqrt{s}=319 \mathrm{GeV}$ with integrated luminosity of $245 \mathrm{pb}^{-1}$ for $e^{+} p$ and $166 \mathrm{pb}^{-1}$ for $e^{-} p$ collisions. No evidence for leptoquark compelled them to exclude second and third generations of leptoquarks with masses under $712 \mathrm{GeV}$ and $479 \mathrm{GeV}$ respectively for couplings to every generation of quark and lepton being $Y=0.3$. Both the surveys present the upper limit on the coupling $Y$ as a function of $M_{\phi}$; moreover, Ref. [43] displays allowed branching of the leptoquark to electron mode with its varying mass. The ZEUS Collaboration [44] also carried out similar analysis and excluded first generation of leptoquark for masses up to $699 \mathrm{GeV}$ with the coupling $Y=0.3$. However, their recent result [45], using high-precision data from HERA with $1 \mathrm{fb}^{-1}$ of integrated luminosity, has pushed the limit in $\mathrm{TeV}$ range; additionally the upper limit on coupling to mass ratio for individual leptoquark has also been put up. Nevertheless, it is interesting to mention that an anomalous measurement at high values of squared four-momentum transfer was observed by both of the H1 [100] and ZEUS [101] Collaborations.

Leptoquarks have been looked for in hadronic colliders as well. The first measurement in this context was done at the CERN $p \bar{p}$ collider Spp̄S. The UA2 detector [46] analysed $13 \mathrm{pb}^{-1}$ data from the CERN Spp̄S collider at $\sqrt{s}=630$ $\mathrm{GeV}$ and set up a lower bound on the mass of first generation leptoquark at $67 \mathrm{GeV}(76 \mathrm{GeV})$ with $95 \%$ C.L. for the

\footnotetext{
${ }_{3}$ As the study suggests, this limit depends on the representation of leptoquark.
} 
branching into the electron mode being $50 \%$ (100\%). The $\mathrm{CDF}$ and $\mathrm{D} \varnothing$ Collaborations have tried extensively to probe the signature of leptoquarks at other $p \bar{p}$ collider, the Fermilab Tevatron. In their final updates, CDF Collaboration $[47,48]$ has worked with data collected from the Fermilab Tevatron collider at $\sqrt{s}=1.96 \mathrm{TeV}$ with integrated luminosities of $203 \mathrm{pb}^{-1}$ and $198 \mathrm{pb}^{-1}$ respectively for pair production of first and second generation of scalar leptoquarks. The lack of any significant signal event led to set the lower bounds on masses of first and second generation scalar leptoquarks being $236 \mathrm{GeV}(205 \mathrm{GeV})$ and $226 \mathrm{GeV}(208 \mathrm{GeV})$ for $\beta=1(0.5)$ respectively at $95 \%$ C.L. The CDF Collaboration [49] have also looked over the Fermilab Tevatron data with $\mathcal{L}_{\text {int }}=322 \mathrm{pb}^{-1}$ in search for a third generation vector leptoquark decaying to a $b$-quark and $\tau$-lepton. Agreement of observation with SM prediction resulted in exclusion of third generation vector leptoquarks below $317 \mathrm{GeV}(251 \mathrm{GeV})$ of mass, assuming Yang-Mills (minimal) couplings. ${ }^{4}$ The D $\varnothing$ Collaboration [50-52] too has looked for pair production of first, second and third generations of scalar leptoquarks separately, based on the data set form the Fermilab Tevatron at $\sqrt{s}=1.96 \mathrm{TeV}$. Due to non-observance of any excess over the SM expectation, they constrain the three generations of leptoquarks to be heavier than $326 \mathrm{GeV}(\beta=0.5), 316$ $\mathrm{GeV}$ (for $\beta=1$ or $270 \mathrm{GeV}$ for $\beta=0.5$ ) and $247 \mathrm{GeV}$ $\left(Q_{\phi}=-1 / 3\right)$ respectively at $95 \%$ C.L.

However, the most stringent bounds on the leptoquarks so far have been provided by the ATLAS and CMS Collaborations, working with $p p$ collisions at the LHC. Using a data set corresponding to $\mathcal{L}_{i n t}=36.1 \mathrm{fb}^{-1}$ and $\sqrt{s}=13 \mathrm{TeV}$, ATLAS Collaboration [54] has tried to probe the pair production of scalar leptoquarks in first and second generation. Absence of any statistically significant evidence for excess over SM allowed for exclusion of first and second generation scalar leptoquarks lighter than $1400 \mathrm{GeV}(1290 \mathrm{GeV})$ and $1560 \mathrm{GeV}(1230 \mathrm{GeV})$ at $95 \%$ C.L. presuming $\beta=1(0.5)$. The same data set has also been used to rule out the third generation of scalar leptoquarks having masses below $800 \mathrm{GeV}$ independent of any branching ratio and masses under $1 \mathrm{TeV}$ for $\beta=0$ or 1 [53]. On the other hand, CMS Collaboration $[55,58]$ has also analysed the LHC data at same $\sqrt{s}$, but with an integrated luminosity of $35.9 \mathrm{fb}^{-1}$ and restricted the first and second generation scalar leptoquark masses to higher than $1435 \mathrm{GeV}(1270 \mathrm{GeV})$ and $1530 \mathrm{GeV}(1285 \mathrm{GeV})$ respectively for $\beta=1(0.5)$ at $95 \%$ C.L. With the help of data collected from LHC at $\sqrt{s}=13 \mathrm{TeV}$ and $\mathcal{L}_{i n t}=35.9 \mathrm{fb}^{-1}$ CMS Collaboration [57,59] has also explored the possibil-

\footnotetext{
${ }^{4}$ Usually, a dimensionless parameter $\kappa\left(\equiv 1-\kappa_{G}\right)$ is introduced while describing the interaction of a vector leptoquark with gluons [102]. It is related to the anomalous chromo-magnetic moment and anomalous chromo-electric dipole moment of the vector leptoquark. The scenario of Yang-Mills coupling is represented by $\kappa=1$ whereas $\kappa=0$ indicates minimal coupling case.
}

ity of pair production for third generation scalar leptoquarks. Due to inadequacy of signal event over SM background, they declare at $95 \%$ C.L. that the third generation scalar leptoquarks decaying to a top-quark and a $\tau$-lepton should have masses greater than $900 \mathrm{GeV}$ and the same decaying to a bottom-quark and a $\tau$-lepton should have masses above 1020 $\mathrm{GeV}$ for $\beta=1$. The CMS Collaboration [56] has looked into the neutrino modes of decay for both scalar and vector leptoquarks too. These invisible modes put strong limit on the vector leptoquarks. If the vector leptoquark has $50 \%$ branching fraction to $t v$ mode and the rest $50 \%$ to $b \tau$ channel, then mass of it below $1530 \mathrm{GeV}(1115 \mathrm{GeV})$ is excluded for $\kappa=1(\kappa=0)$.

Most of the analysis mentioned above assumes the leptoquark to interact with one generation of quark and lepton only. However, when the leptoquark is supposed to have interactions with all generations of quarks and leptons, the branching fraction to different modes get reduced and the bounds thus become more relaxed. In our simulation, we have searched for a $70 \mathrm{GeV}$ leptoquark at HERA, a $900 \mathrm{GeV}$ leptoquark at $\mathrm{LHeC}$ and a $1.5 \mathrm{TeV}(2.0 \mathrm{TeV})$ leptoquark at FCC-he (run II) through the phenomenon of RAZ. For the light leptoquark, we choose the couplings to be significantly smaller than electromagnetic coupling in order to satisfy the cross-section limit by ZEUS Collaboration [103], as shown by the first diagram in Fig. 2. Furthermore, the couplings are adjusted to obey the CDF bounds $[104,105]$ in such a way that branching to the second generation of quark and lepton remains below $23 \%$ and the same to $b \tau$ or $\bar{b} \tau \operatorname{mode}^{5}$ stays under $22 \%$, as depicted by the second and third diagrams in the first row of Fig. 2. For the scalar leptoquarks of mass $900 \mathrm{GeV}$, we pick the couplings respecting the bounds by ATLAS [53] and CMS [55,58] Collaborations coming from charged lepton decay modes since the restrictions from neutrino modes [56] are already satisfied in these cases. These constraints are illustrated in the second row of Fig. 2. On the other hand, for the vector leptoquarks of same mass, we consider the bounds from invisible modes [56] with $\kappa=0$, as shown in the third row of Fig. 2. The theoretical prediction for the cross-section of vector leptoquark pair production in Yang-Mills scenario is so high that it is quite impossible to meet the observed limits of cross-section times branching fraction squared for all the three generations of neutrinos, and therefore, we deliberately omit the $\kappa=1$ case from our consideration. On the other hand, it is easily comprehensible from Fig. 2 that there is effectively no restriction on the branching fractions of scalar and vector leptoquarks beyond mass $1.5 \mathrm{TeV}$. So, we adopt equal coupling for all the allowed interactions in case of $1.5 \mathrm{TeV}(2.0 \mathrm{TeV})$ scalar and vector

\footnotetext{
5 Actually, to obey the CDF plots in Fig. 2, one has to satisfy $\sigma_{p \bar{p} \rightarrow \phi \bar{\phi}} \times$ $\mathcal{B}_{b \tau / \bar{b} \tau}^{2} \leq 20 \mathrm{pb}$ where the symbol $\mathcal{B}$ is used to represent branching fraction throughout the text.
} 

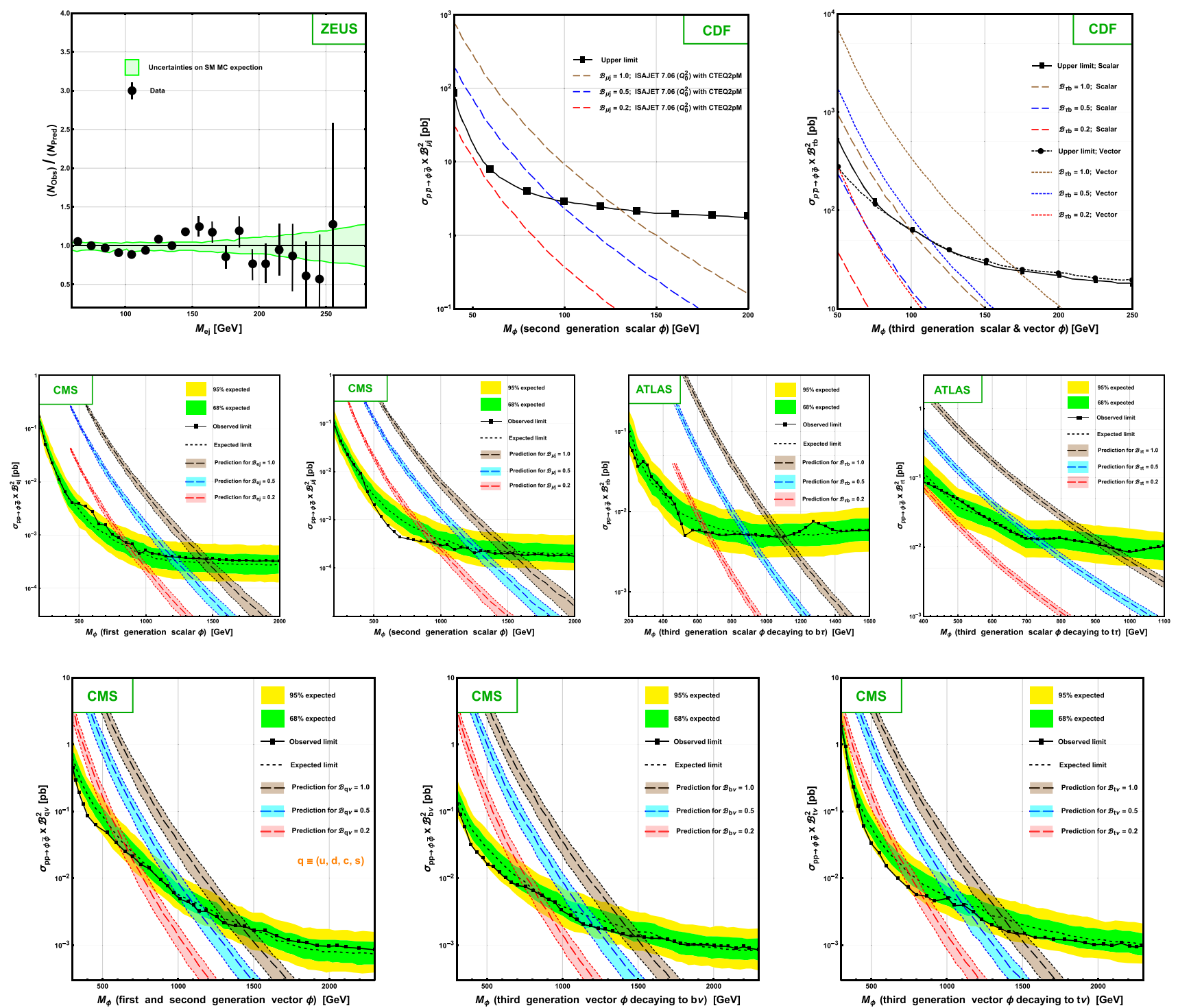

Fig. 2 Different experimental bounds on leptoquarks. The plots in the first row have been used for choosing the benchmark points for $70 \mathrm{GeV}$ leptoquarks, the diagrams in the second row provide bounds on heavier scalar leptoquarks whereas the images in the third row serve the same purpose for heavier vector leptoquarks. In case of vectors, we have taken $\kappa=0$ only

decay modes are not accessible in case BP1 due to scarcity of enough phase space. The production cross-sections for all the leptoquarks associated with a photon are listed in Table 4. Actually, since the hard-scattering processes involve real photon emission the total cross-sections are divergent. ${ }^{6}$ To avoid the singularity, we choose a suitable cut on the transverse momentum of photon $\left(p_{T}^{\gamma}\right)$ being larger than $20 \mathrm{GeV}$ for our entire simulation.

\footnotetext{
${ }^{6}$ Cross-section for any process incorporating real photon emission from massless charged particle suffers from infra-red (IR) and collinear divergences. Though the IR divergence can be controlled by considering a tiny but non-vanishing mass for the radiating charged particle, the collinear divergence still remains.
} 
Table 2 Chosen couplings to different generations of quarks and leptons for various leptoquarks satisfying the experimental bounds discussed in Sect. 3

\begin{tabular}{lllllll}
\hline$\phi$ & $Y_{L}^{11}$ & \multicolumn{1}{c}{$Y_{L}^{22}$} & $Y_{L}^{33}$ & $Y_{R}^{11}$ & $Y_{R}^{22}$ & \multicolumn{1}{l}{$Y_{R}^{33}$} \\
\hline BP1: & $H E R A, M_{\phi}=70 \mathrm{GeV}$ \\
$R_{2}$ & 0.035 & 0.018 & 0.017 & 0.035 & 0.018 & 0.017 \\
$\widetilde{S}_{1}$ & - & - & - & 0.035 & 0.022 & 0.022 \\
$S_{3}$ & 0.035 & 0.022 & 0.022 & - & - & - \\
$V_{2 \mu}$ & 0.035 & 0.022 & 0.022 & 0.035 & 0.022 & 0.022 \\
$\widetilde{U}_{1 \mu}$ & - & - & - & 0.035 & 0.018 & 0.017 \\
$\boldsymbol{U}_{3 \mu}$ & 0.035 & 0.018 & 0.017 & - & - & - \\
$B P 2:$ & $L H e C, M_{\phi}=900 \mathrm{GeV}$ & & & \\
$R_{2}$ & 0.200 & 0.200 & 0.200 & 0.200 & 0.200 & 0.200 \\
$\widetilde{S}_{1}$ & - & - & - & 0.140 & 0.140 & 0.200 \\
$S_{3}$ & 0.140 & 0.140 & 0.200 & - & - & - \\
$V_{2 \mu}$ & 0.100 & 0.100 & 0.200 & 0.200 & 0.200 & 0.200 \\
$\widetilde{U}_{1 \mu}$ & - & - & - & 0.140 & 0.140 & 0.200 \\
$\boldsymbol{U}_{3 \mu}$ & 0.140 & 0.140 & 0.200 & - & - & - \\
$B P 3(B P 4): F C C-I(I I), M$ \\
$B P$
\end{tabular}

\section{Set up for the collider simulation}

As explained in Sect. 2, only six leptoquarks, i.e. $\left(\widetilde{S}_{1}^{+4 / 3}\right)^{c}$, $\left(R_{2}^{+5 / 3}\right)^{c},\left(S_{3}^{+4 / 3}\right)^{c},\left(\tilde{U}_{1 \mu}^{+5 / 3}\right)^{c},\left(V_{2 \mu}^{+4 / 3}\right)^{c}$ and $\left(U_{3 \mu}^{+5 / 3}\right)^{c}$ have zeros within the observable limit in their differential angular distributions while getting produced at $e-p$ collider associated with a photon. In this analysis we reconstruct these leptoquarks via their decay products in the visible channel, i.e. to a charged lepton and a quark. In a process of $e p \rightarrow \phi \gamma$, the final state requires to have at least one hard photon, a hard charged lepton and a quark jet. In order to remove the SM background, we choose the charged lepton to be muon. It is also worth noticing that for $\boldsymbol{S}_{3}^{c}, R_{2}^{c}, V_{2 \mu}^{c}$ and $\boldsymbol{U}_{3 \mu}^{c}$ there are two states which can give rise to the similar decay products, e.g. $\bar{c} \mu$ in case of $\left(R_{2}^{+5 / 3}\right)^{c}$ and $\bar{s} \mu$ in case of $\left(R_{2}^{+2 / 3}\right)^{c}$, while only one of them produces the zero in the angular distribution. To avoid such unwanted model backgrounds we need to separate the jets by reconstructing their charge. We emphasize here again that for models $\widetilde{S}_{1}$ and $\widetilde{U}_{1 \mu}$ no such model background exists.

In this section we will describe the set up for the collider analysis which will be carried out for three different colliders namely HERA [89,90], LHeC and FCC-he [91,92]. For this purpose we first implement the models at SARAH-
Table 3 Branching fractions of different leptoquarks for masses and couplings specified in Table 2. Branching fractions for BP4 scenario have not been displayed explicitly because they differ from BP3 case at third decimal places only

\begin{tabular}{|c|c|c|c|c|c|c|c|}
\hline \multirow{2}{*}{$\begin{array}{l}\text { Decay } \\
\text { modes }\end{array}$} & \multicolumn{3}{|c|}{ Branching fractions } & \multirow{2}{*}{$\begin{array}{l}\text { Decay } \\
\text { modes }\end{array}$} & \multicolumn{3}{|c|}{ Branching fractions } \\
\hline & BP1 & $\mathrm{BP} 2$ & $\mathrm{BP} 3$ & & $\mathrm{BP} 1$ & BP2 & BP3 \\
\hline \multicolumn{4}{|c|}{ Scalar leptoquarks } & \multicolumn{4}{|c|}{ Vector leptoquarks } \\
\hline \multicolumn{4}{|c|}{ Leptoquark $\left(\widetilde{S}_{1}^{+4 / 3}\right)^{c}$} & \multicolumn{4}{|c|}{ Leptoquark $\left(\widetilde{U}_{1}^{+5 / 3}\right)^{c}$} \\
\hline$e d$ & 0.560 & 0.247 & 0.333 & $e \bar{u}$ & 0.791 & 0.254 & 0.335 \\
\hline$\mu s$ & 0.221 & 0.247 & 0.333 & $\mu \bar{c}$ & 0.209 & 0.254 & 0.335 \\
\hline$\tau b$ & 0.219 & 0.505 & 0.333 & $\tau \bar{t}$ & - & 0.491 & 0.331 \\
\hline \multicolumn{4}{|c|}{ Leptoquark $\left(R_{2}^{+5 / 3}\right)^{c}$} & \multicolumn{4}{|c|}{ Leptoquark $\left(V_{2}^{+4 / 3}\right)^{c}$} \\
\hline$e \bar{u}$ & 0.791 & 0.342 & 0.335 & ed & 0.559 & 0.278 & 0.333 \\
\hline$\mu \bar{c}$ & 0.209 & 0.342 & 0.335 & $\mu s$ & 0.221 & 0.278 & 0.333 \\
\hline$\tau \bar{t}$ & - & 0.317 & 0.330 & $\tau b$ & 0.220 & 0.444 & 0.333 \\
\hline \multicolumn{4}{|c|}{ Leptoquark $\left(R_{2}^{+2 / 3}\right)^{c}$} & \multicolumn{4}{|c|}{ Leptoquark $\left(V_{2}^{+1 / 3}\right)^{c}$} \\
\hline$e \bar{d}$ & 0.362 & 0.169 & 0.167 & $e u$ & 0.314 & 0.225 & 0.167 \\
\hline$\mu \bar{s}$ & 0.096 & 0.169 & 0.167 & $\mu c$ & 0.124 & 0.225 & 0.167 \\
\hline$\tau \bar{b}$ & 0.085 & 0.169 & 0.167 & $\tau t$ & - & 0.213 & 0.164 \\
\hline$v_{e} \bar{u}$ & 0.362 & 0.169 & 0.167 & $v_{e} d$ & 0.314 & 0.056 & 0.167 \\
\hline$v_{\mu} \bar{c}$ & 0.096 & 0.169 & 0.167 & $v_{\mu} s$ & 0.124 & 0.056 & 0.167 \\
\hline$v_{\tau} \bar{t}$ & - & 0.156 & 0.165 & $v_{\tau} b$ & 0.123 & 0.225 & 0.167 \\
\hline \multicolumn{4}{|c|}{ Leptoquark $\left(S_{3}^{+4 / 3}\right)^{c}$} & \multicolumn{4}{|c|}{ Leptoquark $\left(U_{3}^{+5 / 3}\right)^{c}$} \\
\hline$e d$ & 0.560 & 0.247 & 0.333 & $e \bar{u}$ & 0.791 & 0.254 & 0.335 \\
\hline$\mu s$ & 0.221 & 0.247 & 0.333 & $\mu \bar{c}$ & 0.209 & 0.254 & 0.335 \\
\hline$\tau b$ & 0.219 & 0.505 & 0.333 & $\tau \bar{t}$ & - & 0.491 & 0.331 \\
\hline \multicolumn{4}{|c|}{ Leptoquark $\left(S_{3}^{+1 / 3}\right)^{c}$} & \multicolumn{4}{|c|}{ Leptoquark $\left(U_{3}^{+2 / 3}\right)^{c}$} \\
\hline$e u$ & 0.314 & 0.126 & 0.167 & $e \bar{d}$ & 0.362 & 0.125 & 0.167 \\
\hline$\mu c$ & 0.124 & 0.126 & 0.167 & $\mu \bar{s}$ & 0.096 & 0.125 & 0.167 \\
\hline$\tau t$ & - & 0.239 & 0.165 & $\tau \bar{b}$ & 0.085 & 0.256 & 0.167 \\
\hline$v_{e} d$ & 0.314 & 0.126 & 0.167 & $v_{e} \bar{u}$ & 0.362 & 0.125 & 0.167 \\
\hline$v_{\mu} s$ & 0.124 & 0.126 & 0.167 & $v_{\mu} \bar{c}$ & 0.096 & 0.125 & 0.167 \\
\hline$v_{\tau} b$ & 0.123 & 0.257 & 0.167 & $v_{\tau} \bar{t}$ & - & 0.242 & 0.165 \\
\hline \multicolumn{4}{|c|}{ Leptoquark $\left(S_{3}^{-2 / 3}\right)^{c}$} & \multicolumn{4}{|c|}{ Leptoquark $\left(U_{3}^{-1 / 3}\right)^{c}$} \\
\hline$v_{e} u$ & 0.717 & 0.257 & 0.335 & $v_{e} \bar{d}$ & 0.667 & 0.247 & 0.333 \\
\hline$v_{\mu} c$ & 0.283 & 0.257 & 0.335 & $v_{\mu} \bar{s}$ & 0.176 & 0.247 & 0.333 \\
\hline$v_{\tau} t$ & - & 0.487 & 0.330 & $v_{\tau} \bar{b}$ & 0.157 & 0.505 & 0.333 \\
\hline
\end{tabular}

4.14.2 [106] and then prepare the model files for CalcHEP [107]. Determination of decay branching fractions for leptoquarks, estimation of production cross-sections associated with a photon and event generation are executed via CalcHEP. It is worth mentioning that cross-section estimation and event generation are done with the help of NNPDF parton distribution functions [108] at renormalization or factorization scale of $\sqrt{\hat{s}}$ where $\hat{s}$ is the parton level centre of momentum energy. For the analysis, we fed the CalcHEP-generated ".lhe" files 
Table 4 Production cross-sections (in fb) of various leptoquarks associated with a photon at different $e-p$ colliders for different benchmark points after applying the cut $p_{T}^{\gamma}>20 \mathrm{GeV}$. As already mentioned ear- lier, $S_{3}^{-2 / 3}$ and $U_{3 \mu}^{-1 / 3}$ can not be produced at $e$ - $p$ collider due to the gauge structure of the Lagrangian

\begin{tabular}{|c|c|c|c|c|c|c|c|c|c|c|}
\hline \multirow[t]{3}{*}{ Collider } & \multicolumn{5}{|c|}{ Scalar leptoquarks } & \multicolumn{5}{|c|}{ Vector leptoquarks } \\
\hline & \multirow{2}{*}{$\begin{array}{l}\text { Singlet } \\
\left(\widetilde{S}_{1}^{+4 / 3}\right)^{c}\end{array}$} & \multicolumn{2}{|l|}{ Doublet } & \multicolumn{2}{|l|}{ Triplet } & \multirow{2}{*}{$\begin{array}{l}\text { Singlet } \\
\left(\widetilde{U}_{1 \mu}^{+5 / 3}\right)^{c}\end{array}$} & \multicolumn{2}{|l|}{ Doublet } & \multicolumn{2}{|l|}{ Triplet } \\
\hline & & $\left(R_{2}^{+5 / 3}\right)^{c}$ & $\left(R_{2}^{+2 / 3}\right)^{c}$ & $\left(S_{3}^{+4 / 3}\right)^{c}$ & $\left(S_{3}^{+1 / 3}\right)^{c}$ & & $\left(V_{2 \mu}^{+4 / 3}\right)^{c}$ & $\left(V_{2 \mu}^{+1 / 3}\right)^{c}$ & $\left(U_{3 \mu}^{+5 / 3}\right)^{c}$ & $\left(U_{3 \mu}^{+2 / 3}\right)^{c}$ \\
\hline BP1: HERA & 21.40 & 5.57 & 8.45 & 21.44 & 57.19 & 5.04 & 76.53 & 199.73 & 5.04 & 7.50 \\
\hline BP2: LHeC & 0.171 & 0.036 & 0.027 & 0.17 & 0.91 & 0.017 & 0.871 & 7.43 & 0.017 & 0.013 \\
\hline BP3: FCC I & 0.934 & 0.067 & 0.047 & 0.93 & 3.8 & 0.066 & 3.7 & 15.1 & 0.066 & 0.045 \\
\hline BP4: FCC II & 2.77 & 0.32 & 0.23 & 2.7 & 8.2 & 0.319 & 10.9 & 32.6 & 0.32 & 0.23 \\
\hline
\end{tabular}

to PYTHIA8 $[109,110]$ in order to simulate the events with FastJET-3.0.3 [111] with following criteria:

1. Although the detector extends up to $|\eta|<4.5$, we are mostly interested in the central events for HERA and therefore consider all the stable particles with $|\eta|<3.5$. However, as the lab frames for LHeC and FCC-he are highly boosted relative to the respective $\mathrm{CM}$ frames, we select $|\eta|<4.5$ for all the stable particles in those two experiments to ensure that the zeros of angular distributions do not lie inside the amputated region.

2. The radius of the jet is chosen to be $R=0.5$, with the following cuts:

- Minimum transverse momentum $p_{T, \min }^{j e t}=20.0$ $\mathrm{GeV}$.

- Jets are reconstructed solely from stable hadrons.

3. The detected stable leptons and photons satisfy the following cuts:

- Minimum transverse momentum $p_{T, \min }=10.0$ $\mathrm{GeV}$.

- Detected leptons are hadronically clean, i.e, hadronic activity within a cone of $\Delta R<0.3$ around each lepton is less than $15 \%$ of the leptonic transverse momentum; they are distinctly registered from the simultaneously produced jets, $\Delta R_{l j}>0.4$; and well distinguished from other stable leptons, if any, $\Delta R_{l l}>0.2$, where $\Delta R_{i j}=\sqrt{\Delta \eta_{i j}^{2}+\Delta \phi_{i j}^{2}}$.

- Detected photons are hadronically clean, i.e, hadronic activity within a cone of $\Delta R<0.2$ around each photon is less than $15 \%$ of the photonic transverse momentum; they are distinctly registered from the simultaneously produced jets, $\Delta R_{\gamma j}>0.2$; and well distinguished from other stable leptons, $\Delta R_{\gamma l}>0.2$.

In the next three sections we discuss the analysis including the angular distributions at HERA, LHeC and FCC-he along with additional cuts.
Table 5 Beam and centre of mass energies along with integrated luminosities at HERA

\begin{tabular}{lllll}
\hline$E_{p}$ & $E_{e^{-}}$ & $\sqrt{s}$ & $\mathcal{L}_{\text {int }}$ & $\mathcal{L}_{\text {int }}^{\text {projected }}$ \\
\hline $920 \mathrm{GeV}$ & $27.5 \mathrm{GeV}$ & $318.1 \mathrm{GeV}$ & $400 \mathrm{pb}^{-1}$ & $100 \mathrm{fb}^{-1}$ \\
\hline
\end{tabular}

\section{The hadron-electron ring accelerator (HERA)}

Motivated by radiation amplitude zero which would give vanishing differential cross-section at some specific zones of phase space, we revisit the hadron-electron ring accelerator (HERA) [89,90] for low mass leptoquarks. We select only those SU(2) singlet, doublet and triplet representations of leptoquarks for which the zeros fall within the observed region of phase space. The analysis follows the prescription described before. HERA originally ran for the centre of momentum energy of $318.12 \mathrm{GeV}$ [90] where electron with energy $27.5 \mathrm{GeV}$ collided with a proton of $920.0 \mathrm{GeV}$ giving rise to an asymmetric collider as shown in Table 5. Each of the H1 and ZEUS experiments collected data with integrated luminosities of around $120 \mathrm{pb}^{-1}$ for $e^{+} p$ and 15 $\mathrm{pb}^{-1}$ for $e^{-} p$ collisions till 2000. Since 2003, an upgraded version of HERA, HERA II, has recorded events with net integrated luminosity of $200 \mathrm{pb}^{-1}$ for both $e^{+} p$ and $e^{-} p$ collisions. For the studies of the zeros of amplitude we project the required luminosity for the $5 \sigma$ signal significance over the total background which includes SM background and the model background both.

In the following subsections we describe different possible scenarios as mentioned in Sect. 3 with the corresponding benchmark points and their collider studies at HERA. As evoked before, each leptoquark $S U(2)_{L}$ multiplet exhibiting RAZ has been presented in the subsequent subsections with respective $S U(3)_{C}, S U(2)_{L}$ representations and $U(1)_{Y}$ charge explicitly mentioned. Since scalar-doublet has two excitation among which one has zero within the observed range of $\cos \theta$ with possible model contamination from the other, we would address that scenario before hand. Here, we 
would also implement the jet-charge reconstruction to reduce such model contamination.

\subsection{Scalar doublet $\boldsymbol{R}_{\mathbf{2}}\left(\mathbf{3}, \mathbf{2}, \frac{7}{3}\right)$}

The scalar doublet leptoquark $R_{2}\left(\mathbf{3}, \mathbf{2}, \frac{7}{3}\right)$ couples with the SM fermions through Yukawa like couplings given by:

$-\mathfrak{L} \supset Y_{L} \bar{u}_{R}\left(i \sigma^{2} R_{2}\right)^{T} \boldsymbol{L}_{L}+Y_{R} \overline{\boldsymbol{Q}}_{L} R_{2} \ell_{R}+$ h.c.

In our notation, $\boldsymbol{Q}_{L}$ and $\boldsymbol{L}_{L}$ are weak isospin doublets for left-handed quarks and leptons, and $u_{R}, d_{R}$ and $l_{R}$ are weak isospin singlets for right-handed up-type quarks, down-type quarks and charged leptons respectively. It is evident from Eq. (8) that both the components of the doublet $R_{2}^{c}$, namely $\left(R_{2}^{+5 / 3}\right)^{c}$ and $\left(R_{2}^{+2 / 3}\right)^{c}$ will be produced at $e$ - $p$ collider simultaneously. However, during their associated production with a photon, the former one displays a zero in its angular distribution within the kinematically allowed region of phase space (to be specific, at $\cos \theta=-\frac{1}{5}$ ) while the later component does not, as shown in Table 1.

Now, both the leptoquarks, produced after $e$ - $p$ collision, will eventually decay to a quark and a lepton. Based on the decay, three types of leptonic final states are possible:

- The first one, leptoquark decays to first generation of quark and lepton. But this channel suffers from enormous irreducible Standard Model background.

- The second one, leptoquark decays to third generation quark and lepton. But, in order to reconstruct the leptoquark in this case, the unstable $\tau$-lepton needs to be reconstructed from its hadronic decay products. This, along with the reconstruction of simultaneously produced top-quark from leptoquark decay will yield several stable jets and increase the combinatorial error. Moreover, reconstruction of top is a difficult task. Also, a $70 \mathrm{GeV}$ leptoquark decaying to a top quark is kinematically forbidden.

- We hence consider the leptoquark decaying into second generation of quark and lepton as shown below:

$$
\begin{aligned}
& e \bar{u} \rightarrow\left(R_{2}^{+5 / 3}\right)^{c} \gamma \rightarrow \mu \bar{c} \gamma, \\
& e \bar{d} \rightarrow\left(R_{2}^{+2 / 3}\right)^{c} \gamma \rightarrow \mu \bar{s} \gamma .
\end{aligned}
$$

It minimizes the SM Background, and the solo challenge remaining is to optimize the signal $\left(R_{2}^{+5 / 3}\right)^{c}$ over the model background $\left(R_{2}^{+2 / 3}\right)^{c}$. During the process of signal background separation, misidentification of $c$ - and $s$-jets can lead to model contamination which can be resolved by determining the charge of the jets as discussed later in this section.
For the collider study, we consider $\left(R_{2}^{+5 / 3}\right)^{c} \rightarrow \mu \bar{c}$ and $\left(R_{2}^{+2 / 3}\right)^{c} \rightarrow \mu \bar{s}$ with the corresponding benchmark point BP1 in Table 2. The branching fractions and production cross-sections associated with a photon $\left(p_{T}^{\gamma} \geq 20 \mathrm{GeV}\right)$ are mentioned in Tables 3 and 4 respectively. In Fig. 3a$\mathrm{c}$, we describe the kinematic distributions for the transverse momenta $\left(p_{T}\right)$ of jet, lepton and photon respectively. It can be seen that jet, lepton and photon are hard enough to satisfy the basic cuts demanded in Sect. 4. One important aspect to notice here is that the distributions for $\left(R_{2}^{+5 / 3}\right)^{c}$ and $\left(R_{2}^{+2 / 3}\right)^{c}$ with respect to the transverse momenta of jet, lepton and photon almost superimpose on each other and it is quite impossible to separate the signatures of these two components of the doublet by applying any cut on any of the transverse momenta.

To determine the four momenta of finalstate particles we consider only the visible decay modes of the leptoquarks, i.e. to muon and quark, which forms jet. Information of the three-momenta of the finalstate particles enables us to determine the boost axis which includes boost along the $z$-axis and in the traverse direction instrumental for reconstructing the CM frame. The RAZ information to distinguish different Leptoquark representations is valid only in the centre of mass frame. The boost effect of the Lab frame can smear or distort such distributions. Here we show for the case of $\left(R_{2}^{+5 / 3}\right)^{c}$ that how the distributions in the lab frame get smeared in comparison with the CM frame and for the rest of the examples we only show the boosted back distributions in the CM frame.

In Fig. 5 we show the angular distribution of the photon with the incoming electron in Lab frame produced in association with $\left(R_{2}^{+5 / 3}\right)^{c}$. As we notice, due to substantial asymmetry of the colliding beams, photons thus produced are highly boosted and are all directed to the opposite direction of the electron beam. With normal bin-width (of 0.04 for the variation of $\cos \theta$ ) which is considered for showing the angular distribution of photon in CM frame, Fig. 5a implies that the zero in distribution gets washed away due to the high boost of the Lab frame. However, with a smaller bin-width of 0.002 in Fig. $5 \mathrm{~b}$ we able to see the RAZ in a different position of $\cos \theta$. We recall here that the position of zero in the angular distribution is independent of the energy of interaction in the CM frame. Thus even though the parton distribution function changes the energy of the parton level interaction, the distribution in the partonic centre of mass frame should remain unaltered. Even so, the observed finalstates in the Lab frame are not in partonic rest frame, rather they are results of e-proton interaction governed by the parton distribution function and boosted along the $\mathrm{z}$-axis. The small transverse boost can be estimated by the uncertainty principle. This causes the smearing in the distributions as can be seen from Fig. 5b, where the RAZ happens around $\cos \theta=-0.99$ in the 


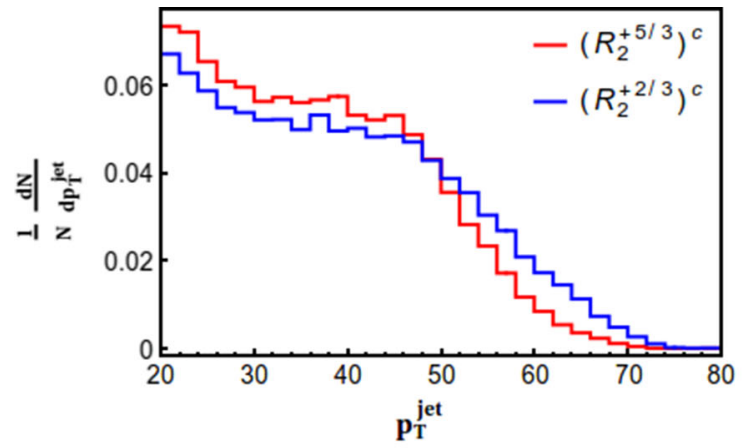

(a)

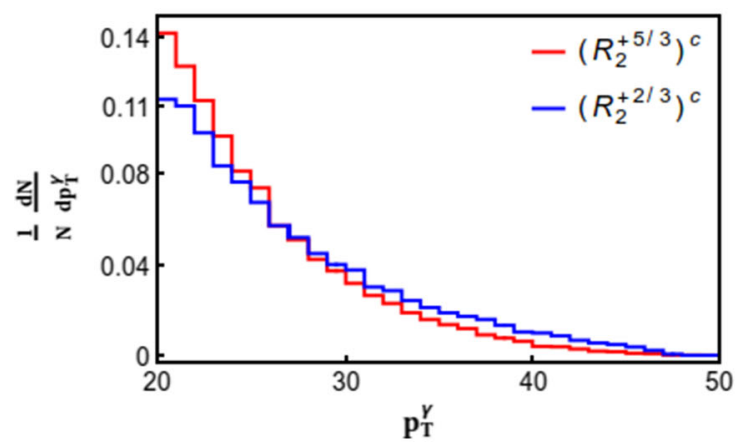

(c)

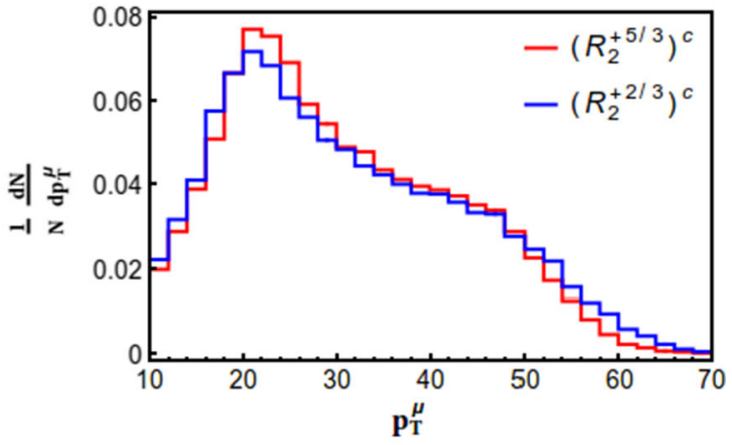

(b)

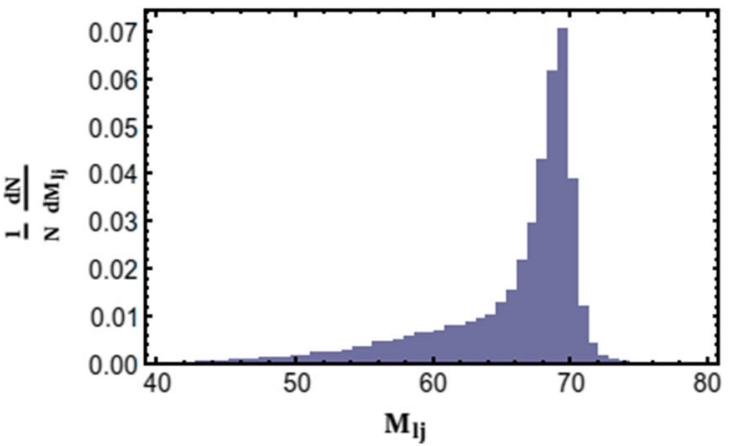

(d)

Fig. $3 p_{T}^{j}, p_{T}^{\ell}, p_{T}^{\gamma}$ and $M_{\ell j}$ distributions at HERA with $\sqrt{s}=318.12 \mathrm{GeV}$ for the leptoquark $R_{2}^{c}$ with mass $70 \mathrm{GeV}$

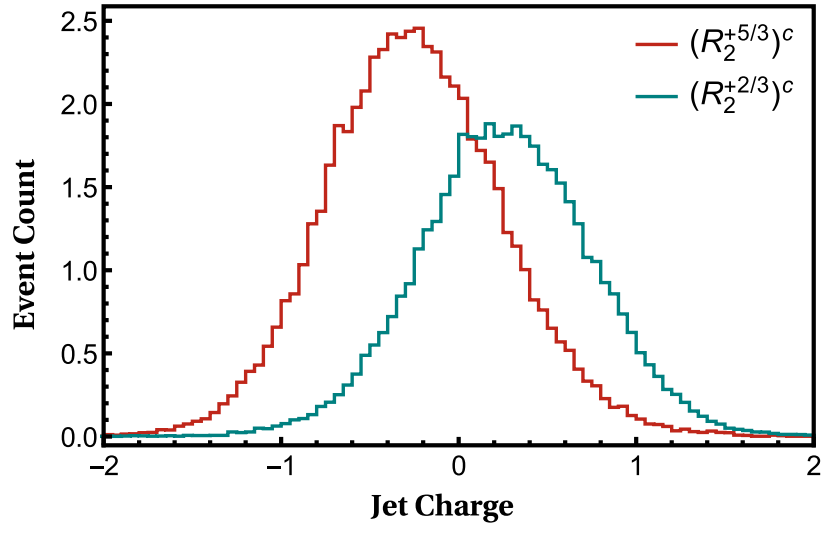

Fig. 4 Determination of the charge (in the unit of $e$ ) for the $c$-and $s$-jets coming from $\left(R_{2}^{+5 / 3}\right)^{c}$ and $\left(R_{2}^{+2 / 3}\right)^{c}$ respectively at HERA

Lab frame, whereas in the reconstructed CM frame the corresponding distribution has RAZ around $\cos \theta=-0.20$ as predicted by the theory. This effect is generic and manifests for all other leptoquarks considered in this study for different collision energies. We henceforth would present only the distributions of photon as observed in boosted-back centre of mass frame.

As already mentioned, the rest frame is reconstructed from tagging the 4-momenta of hard photon, produced in association with a leptoquark which is reconstructed from its decay product of muon and a quark-jet. The event topologies are principally comprised of exactly one hard photon, one hard lepton and jet. Hence the error in boosting back is minimal. We can propose a physical quantity to quantify the error in determining the deep in Lab/CM frame as follows

$\Delta_{\mathrm{Lab} / \mathrm{CM}}=\frac{\left|\zeta_{\mathrm{Lab} / \mathrm{CM}}^{\mathrm{left}}-\zeta_{\mathrm{Lab} / \mathrm{CM}}^{\text {right }}\right|}{\zeta_{\mathrm{Lab} / \mathrm{CM}}^{\mathrm{left}}+\zeta_{\mathrm{Lab} / \mathrm{CM}}^{\text {right }}}$

where $\zeta^{\text {left/right }}$ refers to the number of events within the number (within the minimum and closest peak) of bins in the left/right of the minimum in the respective frames. For the associated production of $\left(R_{2}^{+5 / 3}\right)^{c}$ at HERA with a data set of $100 \mathrm{fb}^{-1}$, we see that the smearing of the boosted minima is around $\sim 61 \%$ in the Lab frame; which is reduced to $\sim 15 \%$ in the reconstructed $\mathrm{CM}$ frame with the expected position of the minima as predicted by the theory.

Next we consider the reconstruction of leptoquark mass via the invariant mass $\left(M_{l j}\right)$ distribution of jet and lepton. For this purpose, we considered each event with at least one muon, one jet and one photon satisfying the above mentioned cuts. We took all possible combinations of jet and lepton to evaluate the invariant mass and plot them in Fig. 3d. The peak around $70 \mathrm{GeV}$ is clearly visible and we select events 


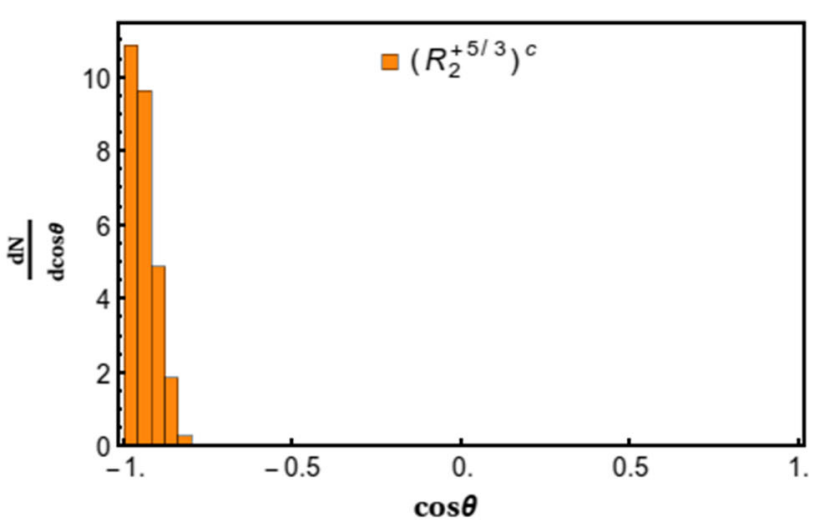

(a) With a bin-width of 0.04

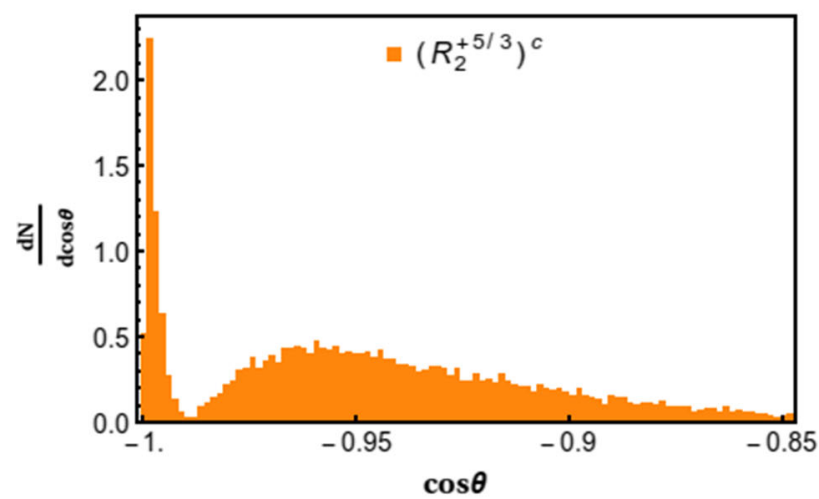

(b) With a bin-width of 0.002

Fig. 5 Distribution of the scattered photon, produced in association with $\left(R_{2}^{+5 / 3}\right)^{c}$ with respect to the angle $(\theta)$ it makes with the incoming electron beam, at $\sqrt{s}=318.12 \mathrm{GeV}$ and $\mathcal{L}_{\text {int }}=100 \mathrm{fb}^{-1}$ in the Lab frame. We present here the same data with varying bin widths. The subfigure a presents the angular distribution with the bin-width of 0.04 for the variation of $\cos \theta$ used in plotting the distribution in CM frame for all cases in this paper. However this makes the signature of amplitude zero washed out due to high boost and we decrease the bin-width to 0.002 in subfigure $\mathbf{b}$ to obtain the manifestation of the amplitude zero, however, shifted due to the boost

within $\pm 10 \mathrm{GeV}$ range of the peak. It is very crucial for the reconstruction of the $\mathrm{CM}$ frame $[86,87]$.

However, as mentioned before $\left(R_{2}^{+5 / 3}\right)^{c}$ and $\left(R_{2}^{+2 / 3}\right)^{c}$ can be indistinguishable in case we cannot separate $c$ - and $s$-jets properly. For this reason we need to determine the charge of the jets from their constituents [112-114]. In Fig. 4 we show the charge distributions of the jets coming from $\left(R_{2}^{+5 / 3}\right)^{c}$ and $\left(R_{2}^{+2 / 3}\right)^{c}$. Though the jet charges are not peaking exactly at $-2 e / 3$ or $e / 3$, it can be observed that the jet charge for the signal, $\left(R_{2}^{+5 / 3}\right)^{c} \rightarrow \mu \bar{c}$, peaks at a negative charge while the model background, $\left(R_{2}^{+2 / 3}\right)^{c} \rightarrow \mu \bar{s}$ reaches the maximum around some positive charge. Both these maxima are wellseparated and hence distinguishable. For our simulation, we have considered the events with jet charge $\leqslant-0.3 e$ to optimise the signal over the model background in case of $R_{2}^{c}$, as shown later (Fig. 5).
Table 6 Number of signal and background events after the cumulative cuts for the scalar doublet leptoquark $R_{2}^{c}$ at $\sqrt{s}=318.12 \mathrm{GeV}$ and $\mathcal{L}_{\text {int }}=100 \mathrm{fb}^{-1}$. Significances with $\mathcal{L}_{i n t}=100 \mathrm{fb}^{-1}$ and $400 \mathrm{pb}^{-1}$ as well as integrated luminosity required for $5 \sigma$ significance at HERA are also estimated

\begin{tabular}{lll}
\hline Cuts & $\begin{array}{l}\text { Signal } \\
\left(R_{2}^{+5 / 3}\right)^{c}\end{array}$ & $\begin{array}{l}\mathrm{SM}+ \\
\left(R_{2}^{+2 / 3}\right)^{c}\end{array}$ \\
\hline $\mathcal{B}\left(R_{2}^{c} \rightarrow \mu \bar{c} / \bar{s}\right): \mathrm{BP} 1$ & & \\
$\geqslant 1 \mu+1 j+1 \gamma$ & 77.2 & 58.5 \\
$\left|M_{\ell j}-M_{R_{2}}\right| \leq 10 \mathrm{GeV}$ & 59.2 & 44.7 \\
$+1 \gamma_{p_{T}>20 \mathrm{GeV}}$ & & \\
$Q_{\text {Jet }}<-0.3$ & 27.7 & \\
$\sigma_{\text {Sig }}\left(\mathcal{L}_{\text {int }}=100 \mathrm{fb}^{-1}\right)$ & 4.8 & \\
$\sigma_{\text {Sig }}\left(\mathcal{L}_{\text {int }}=400 \mathrm{pb}^{-1}\right)$ & 0.3 & \\
$\mathcal{L}_{5 \sigma}\left({\left.\text { in } \mathrm{fb}^{-1}\right)}\right.$ & 108.5 & \\
\hline
\end{tabular}

Once the leptoquark is reconstructed, we determine the cosine of the angle between final state hard photon and the incoming electron beam in the lab frame and then boost it back to the $\mathrm{CM}$ frame of interaction. We impose three additional cuts as mentioned in Table 6, i.e. $(\geqslant 1 \mu+1 j+1 \gamma)+$ $\left(\left|M_{\ell j}-M_{R_{2}}\right| \leq 10 \mathrm{GeV}+1 \gamma_{\mathrm{p}_{\mathrm{T}}>20 \mathrm{GeV}}\right)+\left(\mathrm{Q}_{\mathrm{Jet}}<-0.3\right)$. The final state event numbers after each cumulative cuts for $\left(R_{2}^{+5 / 3}\right)^{c}$ (signal) and $\mathrm{SM}+\left(R_{2}^{+2 / 3}\right)^{c}$ (total background) at $\sqrt{s}=318.12 \mathrm{GeV}$ and $\mathcal{L}_{\text {int }}=100 \mathrm{fb}^{-1}$ are presented in Table 6. Significances with $\mathcal{L}_{i n t}=100 \mathrm{fb}^{-1}$ and $400 \mathrm{pb}^{-1}$ as well as the integrated luminosity required for $5 \sigma$ significance at HERA are also estimated. It can be noticed that a signal significance of $4.8 \sigma$ can be achieved with an integrated luminosity of $100 \mathrm{fb}^{-1}$.

Now, we look for the angular distribution with respect to the angle $(\theta)$ between final state photon and initial state electron in CM frame. Figure 6 illustrates the angular distributions for (a) $\left(R_{2}^{+5 / 3}\right)^{c}$, (b) $\left(R_{2}^{+2 / 3}\right)^{c}$ and (c) the combined one, i.e. $\left(R_{2}^{+5 / 3}\right)^{c}+\left(R_{2}^{+2 / 3}\right)^{c}$, in the CM frame of HERA at $\sqrt{s}=318.12 \mathrm{GeV}$ with an integrated luminosity of 100 $\mathrm{fb}^{-1}$. In Fig. 6a a dip in the angular distribution for $\left(R_{2}^{+5 / 3}\right)^{c}$ is clearly visible, whereas in Fig. $6 \mathrm{~b}$ no such minimum is observed for $\left(R_{2}^{+2 / 3}\right)^{c}$. The combined plot in Fig. 6c still shows the minimum around the same point. However, it should be noticed that the dip is not exactly at $\cos \theta=-\frac{1}{5}$, as mentioned in Table 1, rather it is a bit shifted. The uncertainty in constructing the $\mathrm{CM}$ frame due to parton distribution function is responsible for this minute alteration in the position of RAZ.

\subsection{Scalar Singlet $\tilde{\boldsymbol{S}}_{\mathbf{1}}\left(\overline{\mathbf{3}}, \mathbf{1}, \frac{8}{3}\right)$}

With the scalar singlet leptoquark $\widetilde{S}_{1}\left(\overline{\mathbf{3}}, \mathbf{1}, \frac{8}{3}\right)$, the situation gets much simpler as we have only one excitation and there is no possibility of model contamination. This leptoquark 


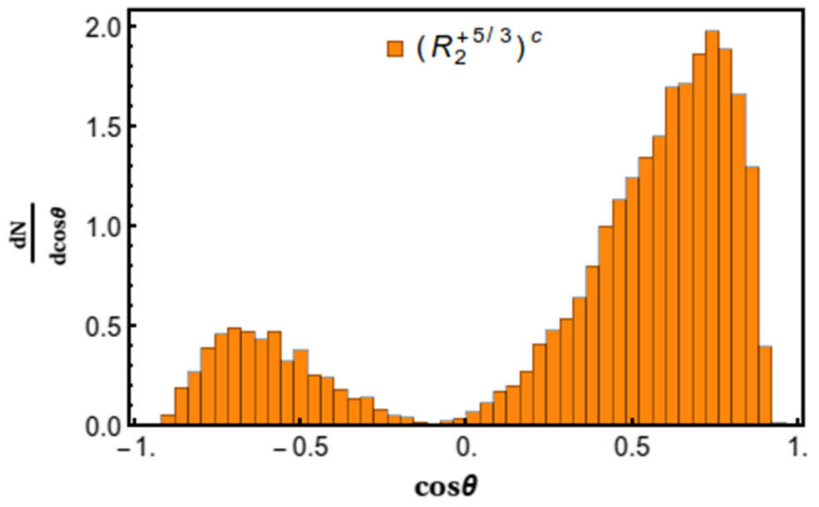

(a) $\left(R_{2}^{+5 / 3}\right)^{c}$

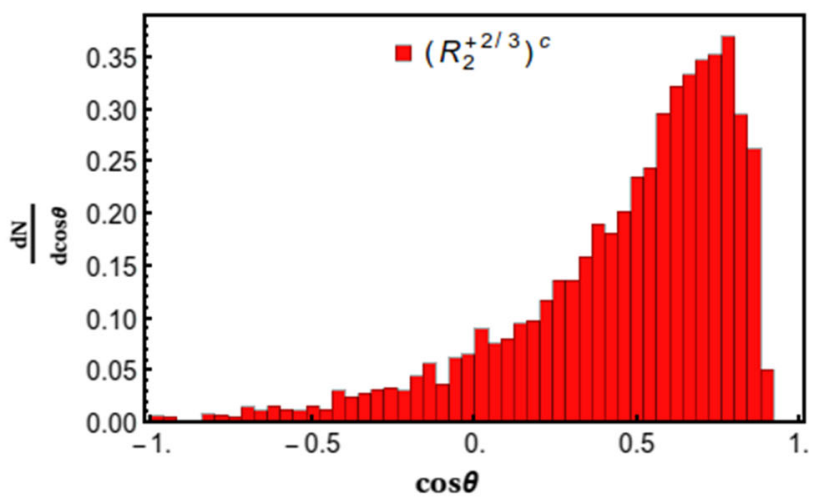

(b) $\left(R_{2}^{+2 / 3}\right)^{c}$

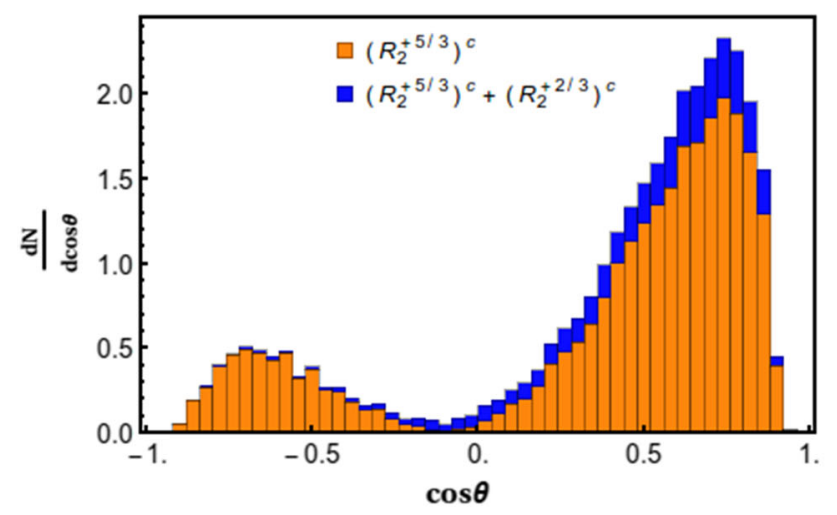

(c) Combined (signal + background)

Fig. 6 Angular distribution with respect to the angle $(\theta)$ made by the photon with the incoming electron beam, at $\sqrt{s}=318.12 \mathrm{GeV}$ and $\mathcal{L}_{\text {int }}=100 \mathrm{fb}^{-1}$ in the $\mathrm{CM}$ frame. The sub-figure (a) shows the angular distribution for the associate production of $\left(R_{2}^{+5 / 3}\right)^{c}$, whereas the subfigure (b) displays the same for $\left(R_{2}^{+2 / 3}\right)^{c}$. In the sub-figure (c), we present the angular distributions of the signal and background together in the rest frame of interaction

couples to SM fermions through Yukawa like coupling given by,

$-\mathfrak{L} \supset Y_{R} \bar{d}_{R}^{c} \widetilde{S}_{1} \iota_{R}+$ h.c.

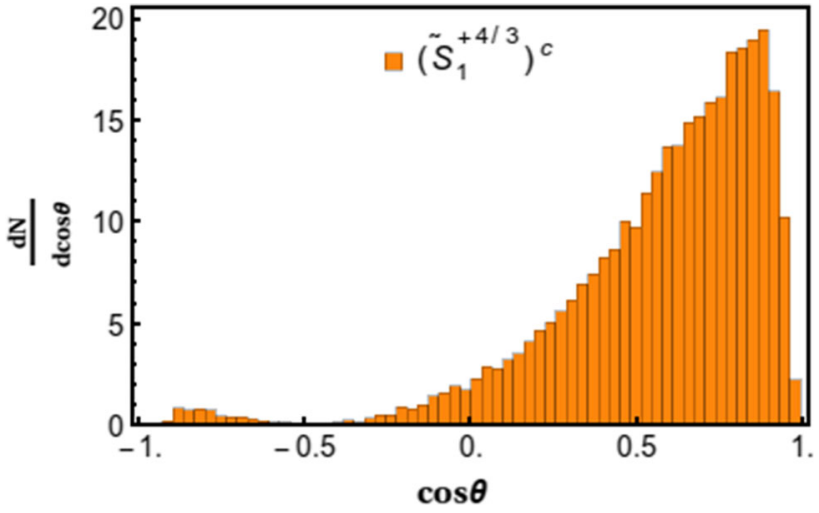

Fig. 7 Angular distribution for the associated production of reconstructed $70 \mathrm{GeV}$ leptoquark $\widetilde{S}_{1}^{c}$ relative to photon angle $(\theta)$ made with electron beam, at $\sqrt{s}=318.12 \mathrm{GeV}$ and $\mathcal{L}_{i n t}=100 \mathrm{fb}^{-1}$ in the rest frame of interaction

When this leptoquark is produced in association with a photon during the $e-p$ collision, its differential distribution with respect to the angle $(\theta)$ made by the photon with the incoming electron beam should exhibit a zero at $\cos \theta=-\frac{1}{2}$. Similar to the previous case, here also we concentrate in the final state invoking muon in order to eliminate the SM backgrounds. The benchmark point (BP1) for the simulation is described in Table 2. The branching fractions and the production crosssections associated with a photon $\left(p_{T}^{\gamma} \geq 20 \mathrm{GeV}\right)$ for the study at HERA are given in Tables 3 and 4 respectively.

Unlike the doublet case, here we have only one excitation leading to the final state topology in electron-hadron collision which constitutes of a muon, a $s$-quark and a photon:

$e p \rightarrow\left(\widetilde{S}_{1}^{+4 / 3}\right)^{c} \gamma \rightarrow \mu s \gamma$.

Similar to the doublet leptoquark case, here also we reconstruct the scalar leptoquark mass via the invariant mass reconstruction of $\mu$-jet in order to go back to the CM frame. The angular distribution at the CM frame of HERA with $\mathcal{L}_{i n t}=$ $100 \mathrm{fb}^{-1}$ relative to the cosine of the angle between the hard photon and incoming electron for the events with exactly one photon with $p_{T}^{\gamma} \geq 20 \mathrm{GeV}$ and exactly one reconstructed leptoquark is shown in Fig. 7. The minimum at $\cos \theta=-\frac{1}{2}$ is clearly visible. The numbers of events ${ }^{7}$ after each cuts for the above mentioned final states at an integrated luminosity of $100 \mathrm{fb}^{-1}$ are tabulated in Table 7 .

\subsection{Scalar triplet $S_{\mathbf{3}}\left(\overline{\mathbf{3}}, \mathbf{3}, \frac{2}{3}\right)$}

We now consider the scalar triplet leptoquark, $\boldsymbol{S}_{3}\left(\overline{\mathbf{3}}, \mathbf{3}, \frac{2}{3}\right)$ for our analysis. This leptoquark couples to the SM fermions

\footnotetext{
7 Since this is a zero-background scenario, we do not mention the significance.
} 
Table 7 Number of events after the cumulative cuts for the scalar singlet leptoquark $\widetilde{S}_{1}^{c}$ and SM background at $\sqrt{s}=318.12 \mathrm{GeV}$ with $\mathcal{L}_{i n t}=$ $100 \mathrm{fb}^{-1}$ at HERA

\begin{tabular}{lll}
\hline Cuts & Signal $\left(\widetilde{S}_{1}^{+4 / 3}\right)^{c}$ & Background \\
\hline $\mathcal{B}\left(\widetilde{S}_{1}^{c} \rightarrow \mu s\right): \mathrm{BP} 1$ & & \\
$\geqslant 1 \mu+1 j+1 \gamma$ & 326.7 & 0.0 \\
$\left|M_{l j}-M_{\widetilde{S}_{1}}\right| \leq 10 \mathrm{GeV}$ & 267.9 & 0.0 \\
$+1 \gamma_{\left(p_{T}>20 \mathrm{GeV}\right)}$ & 263.5 & 0.0 \\
\hline
\end{tabular}

through Yukawa like coupling given by,

$-\mathfrak{L} \supset Y_{L} \overline{\boldsymbol{Q}}_{L}^{c}\left(i \sigma^{2} S_{3}^{a d j}\right) \boldsymbol{L}_{L}+$ h.c.,

where, $S_{3}^{a d j}=\left(\begin{array}{cc}\frac{S_{3}^{+1 / 3}}{\sqrt{2}} & S_{3}^{+4 / 3} \\ S_{3}^{-2 / 3} & -\frac{S_{3}^{+1 / 3}}{\sqrt{2}}\end{array}\right)$ signifies the triplet in the adjoint representation.

Of the three members of the triplet, only two, namely $\left(S_{3}^{+4 / 3}\right)^{c}$ and $\left(S_{3}^{+1 / 3}\right)^{c}$, can be produced from $e$ - $p$ collision, since $S_{3}^{-2 / 3}$ does not couple to electron at all, as can be realised from Eq. (13). Again, only $\left(S_{3}^{+4 / 3}\right)^{c}$ shows RAZ at $\cos \theta=$ $-\frac{1}{2}$ in CM frame whereas $\left(S_{3}^{+1 / 3}\right)^{c}$ does not and acts as model contamination. Following the same argument as before, we look for the leptoquark decaying to second generation lepton and quark, i.e $\mu$ and $s / c$. The interaction at the $e-p$ collider for the associated production with a photon and the decay of these leptoquarks are shown below:

$e p \rightarrow\left(S_{3}^{+4 / 3}\right)^{c} \gamma \rightarrow \mu s \gamma$,

$e p \rightarrow\left(S_{3}^{+1 / 3}\right)^{c} \gamma \rightarrow \mu c \gamma$.

We follow the same approach for event generation and collider simulation with the allowed benchmark point (BP1) in Table 2, the branching fractions in Table 3 and the crosssections associated with a photon $\left(p_{T}^{\gamma} \geq 20 \mathrm{GeV}\right)$ in Table 4 .

We see that while decaying, the signal leptoquark $\left(S_{3}^{+4 / 3}\right)^{c}$ produces a $c$-jet, whereas the background leptoquark $\left(S_{3}^{+1 / 3}\right)^{c}$ gives rise to a $s$-jet. So, we determine the jet-charge in order to separate the final states in a similar fashion as was done for scalar doublet $R_{2}^{c}$ in Sect. 5.1. From Fig. 8 one can notice that the jets from the decay of $\left(S_{3}^{+4 / 3}\right)^{c}$ peaks at a negative charge whereas the same from $\left(S_{3}^{+1 / 3}\right)^{c}$ attains the maximum around some positive charge. Although the peaks of jet-charges are not exactly at $2 e / 3$ or $-e / 3$, they are well-separated and hence can be recognized. For our analysis of $S_{3}^{c}$, we impose a cut and accept the events for which the jet-charge are negative, so that the signal can be optimized over the background.

After the leptoquark mass reconstruction from $\mu$-jet invariant mass, as explained before, we now determine the

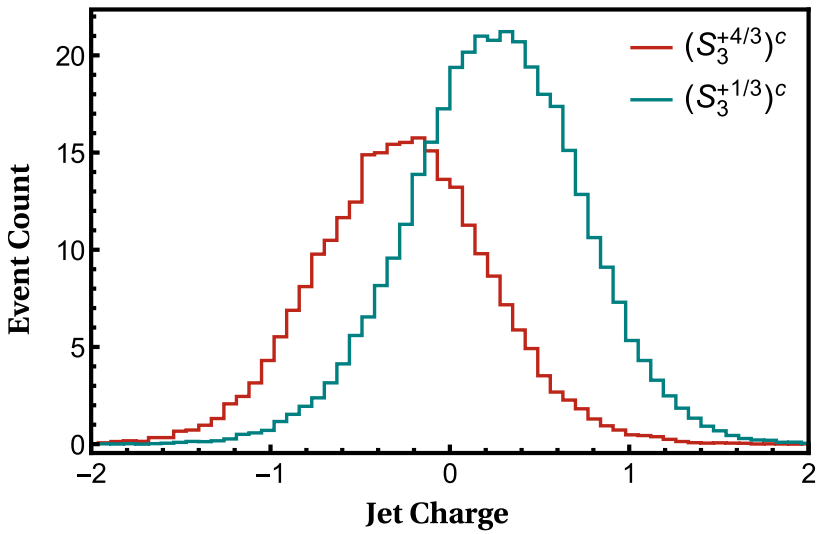

Fig. 8 Reconstructed charge (in the unit of $e$ ) of $c / s$-jets coming from the scalar triplet leptoquark decay

Table 8 Number of events after the cumulative cuts for the scalar triplet leptoquark $\boldsymbol{S}_{3}^{c}$ with $\sqrt{s}=318.12 \mathrm{GeV}$ and $\mathcal{L}_{\text {int }}=100 \mathrm{fb}^{-1}$. Significances with $\mathcal{L}_{i n t}=100 \mathrm{fb}^{-1}$ and $400 \mathrm{pb}^{-1}$ as well as integrated luminosity required for $5 \sigma$ significance at HERA are also estimated

\begin{tabular}{lll}
\hline Cuts & $\begin{array}{l}\text { Signal } \\
\left(S_{3}^{+4 / 3}\right)^{c}\end{array}$ & $\begin{array}{l}\mathrm{SM}+ \\
\left(S_{3}^{+1 / 3}\right)^{c}\end{array}$ \\
\hline $\mathcal{B}\left(\boldsymbol{S}_{3}^{c} \rightarrow \mu s / c\right): \mathrm{BP} 1$ & & \\
$\geqslant 1 \mu+1 j+1 \gamma$ & 328.5 & 520.2 \\
$\left|M_{\ell j}-M_{S_{3}}\right| \leq 10 \mathrm{GeV}$ & 263.3 & 359.6 \\
$+1 \gamma_{p_{T}>20 \mathrm{GeV}}$ & & \\
$Q_{\text {Jet }}<0.0$ & 180.5 & \\
$\sigma_{\text {Sig }}\left(\mathcal{L}_{\text {int }}=100 \mathrm{fb}^{-1}\right)$ & 10.7 & \\
$\sigma_{\text {Sig }}\left(\mathcal{L}_{\text {int }}=400 \mathrm{pb}^{-1}\right)$ & 0.67 & \\
$\mathcal{L}_{5 \sigma}\left(\right.$ in fb $\left.^{-1}\right)$ & 21.8 & \\
\hline
\end{tabular}

cosine of the angle of the hard photon with the incoming electron beam $(\cos \theta)$ in the $\mathrm{CM}$ frame for the final state $\geq 1 \mu+1 j+1 \gamma$. In Table 8 , we tabulate the events coming from $\left(S_{3}^{+4 / 3}\right)^{c}$ and SM processes plus $\left(S_{3}^{+1 / 3}\right)^{c}$ at different stages of imposed cuts for $\mathcal{L}_{\text {int }}=100 \mathrm{fb}^{-1}$. We determine the signal significance at integrated luminosities of $400 \mathrm{pb}^{-1}$ (HERA) and $100 \mathrm{fb}^{-1}$ and also predict the $\mathcal{L}_{\text {int }}$ required for a signal significance of $5 \sigma$. It is evident that the cuts substantially reduce the model background from the production of $\left(S_{3}^{+1 / 3}\right)^{c}$, whereas there is no SM background to be observed.

After implementing the cuts of Table 8, we plot the angular distributions for the leptoquark $\boldsymbol{S}_{3}^{c}$ in Fig. 9. Figure 9(a) clearly shows a zero around $\cos \theta=-\frac{1}{2}$ for $\left(S_{3}^{+4 / 3}\right)^{c}$, while Fig. $9 \mathrm{~b}$ depicts no such zero for $\left(S_{3}^{+1 / 3}\right)^{c}$ in the angular distribution. Like the scalar doublet case, here also the zero is shifted from its theoretical position. We combine the signal and background together in Fig. 9c since both the components with same mass will be produced as parts of the triplet. As can be seen, the zero around $\cos \theta=-\frac{1}{2}$ is gone in case of combined plot, however, a dip is still noticeable. The anal- 


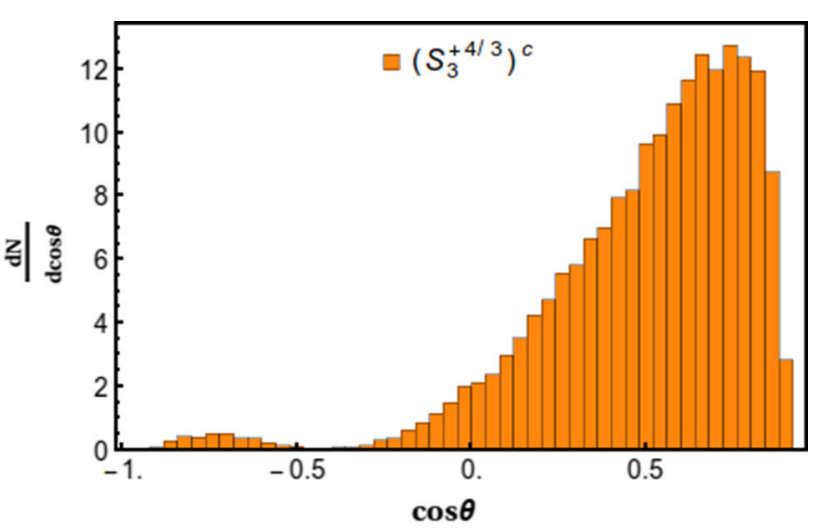

(a) $\left(S_{3}^{+4 / 3}\right)^{c}$

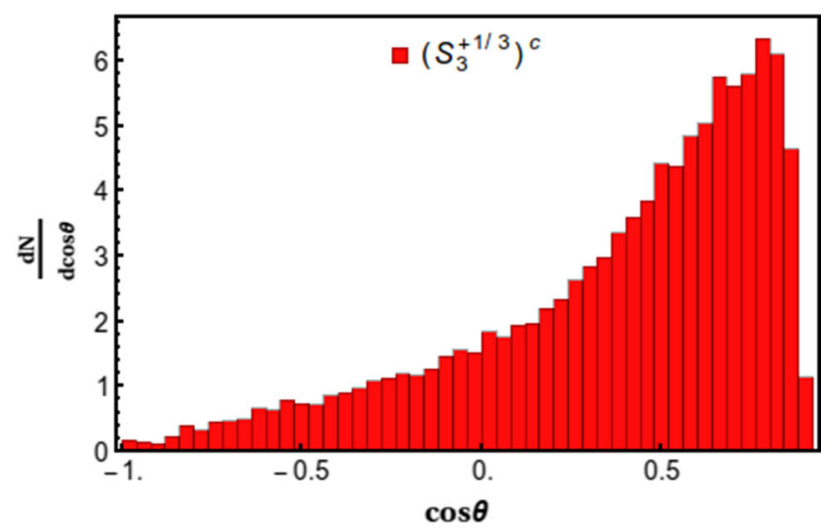

(b) $\left(S_{3}^{+1 / 3}\right)^{c}$

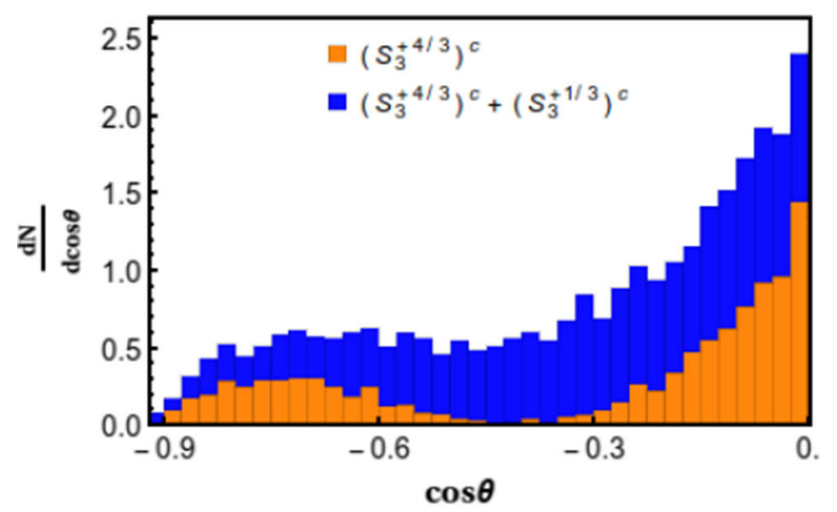

(c) Combined

Fig. 9 Angular distribution for the associated production of reconstructed $70 \mathrm{GeV}$ scalar triplet leptoquark $\boldsymbol{S}_{3}^{c}$ relative to the photon angle $(\theta)$ with electron beam, at $\sqrt{s}=318.12 \mathrm{GeV}$ and $\mathcal{L}_{\text {int }}=100 \mathrm{fb}^{-1}$ in $\mathrm{CM}$ frame. The sub-figure a shows the angular distribution for $\left(S_{3}^{+4 / 3}\right)^{c}$, b shows the same for $\left(S_{3}^{+1 / 3}\right)^{c}$, and $\mathbf{c}$ depicts the combined distribution in the CM frame of interaction

ysis shows that a very early data of $\sim 21.8 \mathrm{fb}^{-1}$ of integrated luminosity can probe the minimum in the angular distribution of photon for the scalar triplet leptoquark $\boldsymbol{S}_{3}^{c}$.

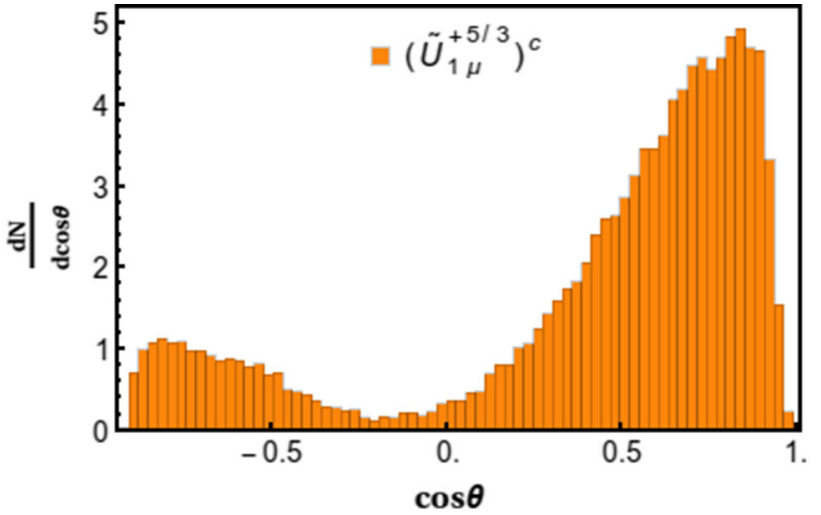

Fig. 10 Angular distribution for the associated production of the reconstructed $70 \mathrm{GeV}$ vector singlet leptoquark $\widetilde{U}_{1 \mu}^{c}$ at $\sqrt{s}=318.12$ $\mathrm{GeV}$ and $\mathcal{L}_{i n t}=100 \mathrm{fb}^{-1}$ at the rest frame of interaction

\subsection{Vector singlet $\tilde{U}_{\mathbf{1} \mu}\left(\mathbf{3}, \mathbf{1}, \frac{10}{3}\right)$}

Having dealt with all the viable models of scalar leptoquarks, exhibiting zeros in their angular distributions, we now address the vector counterparts. We begin our discussion with the vector singlet $\widetilde{U}_{1 \mu}\left(\mathbf{3}, \mathbf{1}, \frac{10}{3}\right)$. It couples with SM fermion through Yukawa like coupling given by,

$-\mathfrak{L} \supset Y_{R} \bar{u}_{R} \gamma^{\mu} \widetilde{U}_{1 \mu} \ell_{R}+$ h.c.

Here we have only one excitation, i.e. $\widetilde{U}_{1 \mu}$. This leptoquark, when gets produced in association with a photon from $e-p$ collision, exhibits zero at $\cos \theta=-\frac{1}{5}$.

For collider study at HERA, we benchmark the scenario by BP1 in Tables 2, 3 and 4. Following the reasoning in the preceding sections, we consider the leptoquark decay to only second generation lepton and quark. After $e-p$ collision, this singlet will be produced in association with a photon and then decay to the desired fermions as follows:

$e p \rightarrow\left(\widetilde{U}_{1 \mu}^{+5 / 3}\right)^{c} \gamma \rightarrow \mu \bar{c} \gamma$

We simulated the events in PYTHIA8 and reconstitute the leptoquark via $c \mu$ invariant mass as before. The angular distribution in the CM frame with respect to the cosine of the angle made by the hard photon with the incoming electron for the events with $(\geqslant 1 \mu+1 j+1 \gamma)+\left(\left|M_{\ell j}-M_{\widetilde{U}_{1 \mu}}\right| \leq\right.$ $\left.10 \mathrm{GeV}+1 \gamma_{\mathrm{p}_{\mathrm{T}}>20 \mathrm{GeV}}\right)$ is plotted in Fig. 10. The existence of a minimum surrounding $\cos \theta=-0.2$ is patently perceptible. The corresponding events ${ }^{8}$ at an integrated luminosity of $100 \mathrm{fb}^{-1}$ are then listed in Table 9 with the cumulative cuts. The number of events seems very promising with an early data in the absence of any SM background.

\footnotetext{
${ }^{8}$ Like the scalar singlet case, here also we do not mention the significance, since it is a zero-background scenario.
} 
Table 9 Number of events after the cumulative cuts for the vector singlet leptoquark $\widetilde{U}_{1 \mu}^{c}$ with $\sqrt{s}=318 \mathrm{GeV}$ and $\mathcal{L}_{i n t}=100 \mathrm{fb}^{-1}$ at HERA

\begin{tabular}{lll}
\hline Cuts & $\operatorname{Signal}\left(\widetilde{U}_{1}^{+5 / 3}\right)^{c}$ & Background \\
\hline $\mathcal{B}\left(\widetilde{U}_{1 \mu}^{c} \rightarrow \mu^{-} \bar{c}\right): \mathrm{BP} 1$ & & \\
$\geqslant 1 \mu+1 j+1 \gamma$ & 74.8 & 0.0 \\
$\left|M_{l j}-M_{\widetilde{U}_{1 \mu}}\right| \leq 10 \mathrm{GeV}$ & 54.9 & 0.0 \\
$+1 \gamma_{\left(p_{T}>20 \mathrm{GeV}\right)}$ & 54.2 & 0.0 \\
\hline
\end{tabular}

\subsection{Vector doublet $\boldsymbol{V}_{\mathbf{2} \boldsymbol{\mu}}\left(\overline{\mathbf{3}}, \mathbf{2}, \frac{5}{3}\right)$}

We next take the vector doublet leptoquark $V_{2 \mu}\left(\overline{\mathbf{3}}, \mathbf{2}, \frac{5}{3}\right)$. This leptoquark couples with the SM fermions through Yukawa like coupling given by,

$$
\begin{aligned}
-\mathfrak{L} \supset Y_{L} \bar{d}_{R}^{c} \gamma^{\mu}\left(i \sigma^{2} V_{2 \mu}\right)^{T} \boldsymbol{L}_{L}+Y_{R} \overline{\boldsymbol{Q}}_{L}^{c} \gamma^{\mu} \\
\times\left(i \sigma^{2} V_{2 \mu}\right) \mathcal{C}_{R}+\text { h.c. }
\end{aligned}
$$

In this case, $\left(V_{2 \mu}^{+4 / 3}\right)^{c}$, the member with isospin projection $+\frac{1}{2}$, shows zero in angular distribution about $\cos \theta=$ $-\frac{1}{2}$ at $\mathrm{CM}$ frame, while $\left(V_{2 \mu}^{+1 / 3}\right)^{c}$ exhibits a monotonically increasing graph of angular distribution with $\cos \theta$. The couplings, decay branching fractions and cross-sections $\left(p_{T}^{\gamma} \geq\right.$ $20 \mathrm{GeV}$ ) are given in Tables 2, 3 and 4 respectively.

The production channel associated with a photon and the decay final sates for $\left(V_{2 \mu}^{+4 / 3}\right)^{c}$ and $\left(V_{2 \mu}^{+1 / 3}\right)^{c}$ at $e-p$ collider are given below:

$\begin{aligned} e p & \rightarrow\left(V_{2 \mu}^{+4 / 3}\right)^{c} \gamma \rightarrow \mu s \gamma \\ e p & \rightarrow\left(V_{2 \mu}^{+1 / 3}\right)^{c} \gamma \rightarrow \mu c \gamma .\end{aligned}$

Similarly to previous cases, here also we determine the charge of the jets from the distributions given below in Fig. 11. We see in Table 10 that a demand of $Q_{\text {Jet }}<-0.3$ reduces the model contamination of $\left(V_{2 \mu}^{+1 / 3}\right)^{c}$ substantially.

Equipped with all the cuts we then reconstruct the leptoquark mass from the $j \mu$ invariant mass distribution as explained before and plot the angular distribution of the hard photon with the incoming electron axis in the $\mathrm{CM}$ frame for the final state with $\geqslant 1 \mu+1 j+1 \gamma$. The angular distributions for $\left(V_{2 \mu}^{+4 / 3}\right)^{c},\left(V_{2 \mu}^{+1 / 3}\right)^{c}$ and the combined scenario are depicted in Fig. 12 by the sub-figures (a), (b) and (c) respectively. It is apparent that for $\left(V_{2 \mu}^{+4 / 3}\right)^{c}$ we see a zero in the neighbourhood of $\cos \theta=-\frac{1}{2}$ which is still reflected in the combined plot as a minimum of the distribution.

The number of events for such final states with cumulative cuts are given in Table 10 at integrated luminosity of $400 \mathrm{pb}^{-1}$ and $100 \mathrm{fb}^{-1}$. It can be seen a very early data of $\sim 8 \mathrm{fb}^{-1}$ can give us a $5 \sigma$ signal significance.

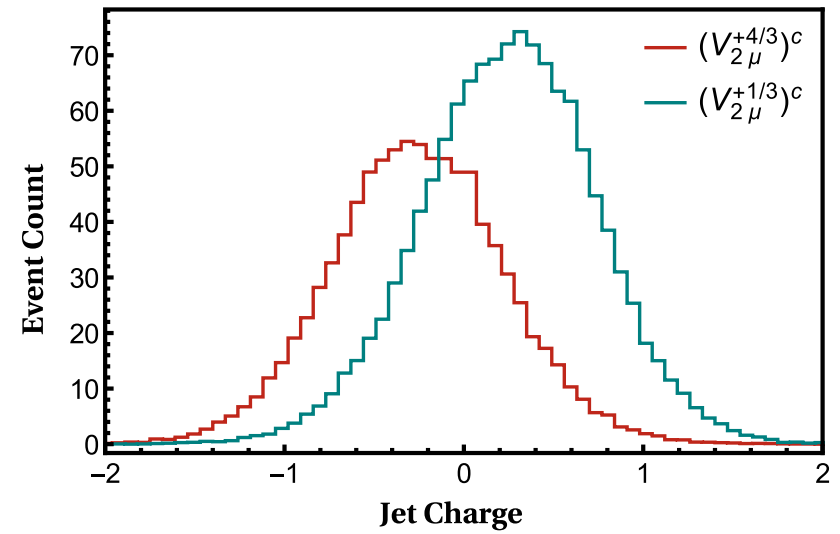

Fig. 11 Charge (in the unit of $e$ ) reconstruction of $c$ - and $s$-jets from the vector doublet leptoquark decay

Table 10 Number of events after the cumulative cuts for the vector doublet leptoquark $V_{2 \mu}^{c}$ at $\sqrt{s}=318.12 \mathrm{GeV}$ and $\mathcal{L}_{\text {int }}=100 \mathrm{fb}^{-1}$. Significances with $\mathcal{L}_{\text {int }}=100 \mathrm{fb}^{-1}$ and $400 \mathrm{pb}^{-1}$ as well as integrated luminosity required for $5 \sigma$ significance at HERA are also estimated

\begin{tabular}{lll}
\hline Cuts & $\begin{array}{l}\text { Signal } \\
\left(V_{2 \mu}^{+4 / 3}\right)^{c}\end{array}$ & $\begin{array}{l}\mathrm{SM}+ \\
\left(V_{2 \mu}^{+1 / 3}\right)^{c}\end{array}$ \\
\hline $\mathcal{B}\left(V_{2 \mu}^{c} \rightarrow \mu s / c\right): \mathrm{BP} 1$ & & \\
$\geqslant 1 \mu+1 j+1 \gamma$ & 1167.6 & 1818.5 \\
$+\left|M_{l j}-M_{V_{2 \mu}}\right| \leq 10 \mathrm{GeV}$ & 929.8 & 1257.8 \\
$+1 \gamma_{p_{T}>20 \mathrm{GeV}}$ & & \\
$Q_{\text {Jet }}<-0.3$ & 435.5 & \\
$\sigma_{\text {Sig }}\left(\mathcal{L}_{\text {int }}=100 \mathrm{fb}^{-1}\right)$ & 17.9 & \\
$\sigma_{\text {Sig }}\left(\mathcal{L}_{\text {int }}=400 \mathrm{pb}^{-1}\right)$ & 1.1 & \\
$\mathcal{L}_{5 \sigma}\left(\mathrm{in} \mathrm{fb}^{-1}\right)$ & 7.8 & \\
\hline
\end{tabular}

\subsection{Vector triplet $\boldsymbol{U}_{\mathbf{3} \boldsymbol{\mu}}\left(\mathbf{3}, \mathbf{3}, \frac{4}{3}\right)$}

We finally consider the last model for our analysis, the vector triplet leptoquark $\boldsymbol{U}_{3 \mu}\left(\mathbf{3}, \mathbf{3}, \frac{4}{3}\right)$. Two out of three members of the triplet, with isospin projections +1 and 0 , can be produced at $e-p$ collider. This leptoquark couples with the SM fermions through Yukawa like coupling given by,

$-\mathfrak{L} \supset Y_{L} \overline{\boldsymbol{Q}}_{L} \gamma^{\mu} U_{3 \mu}^{\text {adj }} \boldsymbol{L}_{L}+$ h.c.,

where, $U_{3 \mu}^{a d j}=\left(\begin{array}{cc}\frac{U_{3 \mu}^{+2 / 3}}{\sqrt{2}} & U_{3 \mu}^{+5 / 3} \\ U_{3 \mu}^{-1 / 3} & -\frac{U_{3 \mu}^{+2 / 3}}{\sqrt{2}}\end{array}\right)$ symbolizes the triplet in adjoint representation.

The interaction at $e-p$ collider for the production and decay of these leptoquarks are,

$e p \rightarrow\left(U_{3 \mu}^{+5 / 3}\right)^{c} \gamma \rightarrow \mu \bar{c} \gamma$

$e p \rightarrow\left(U_{3 \mu}^{+2 / 3}\right)^{c} \gamma \rightarrow \mu \bar{s} \gamma$. 


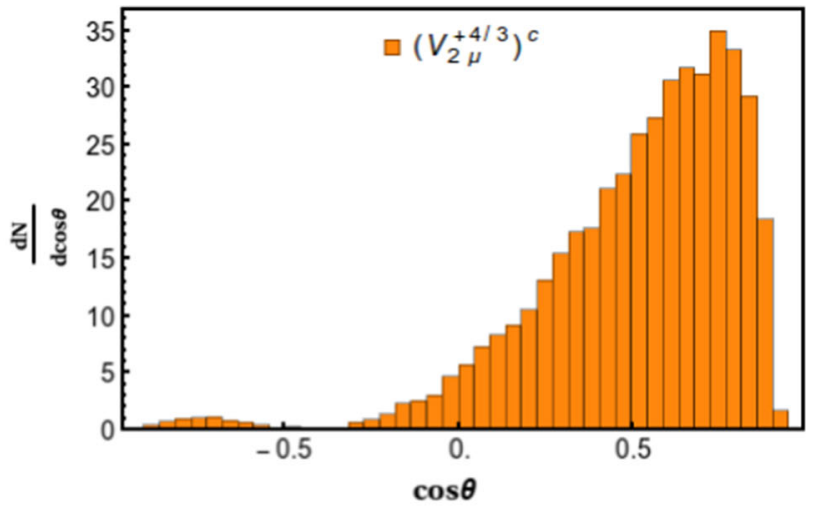

(a) $\left(V_{2 \mu}^{+4 / 3}\right)^{c}$

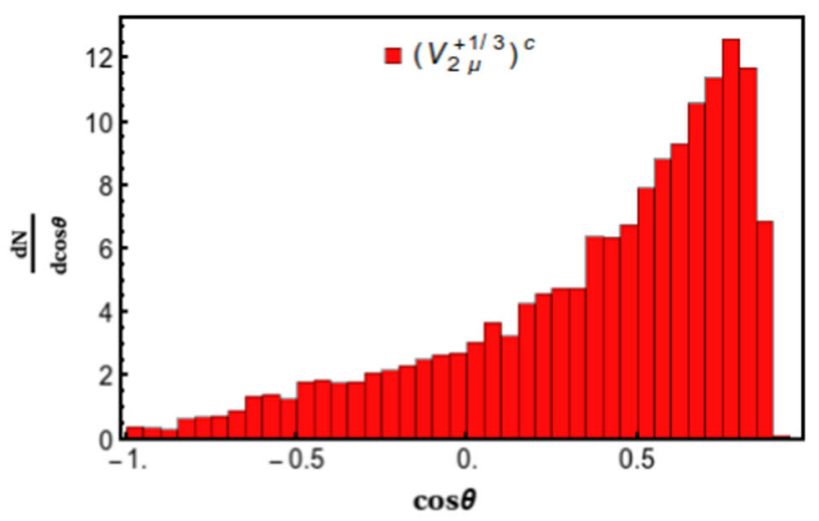

(b) $\left(V_{2 \mu}^{+1 / 3}\right)^{c}$

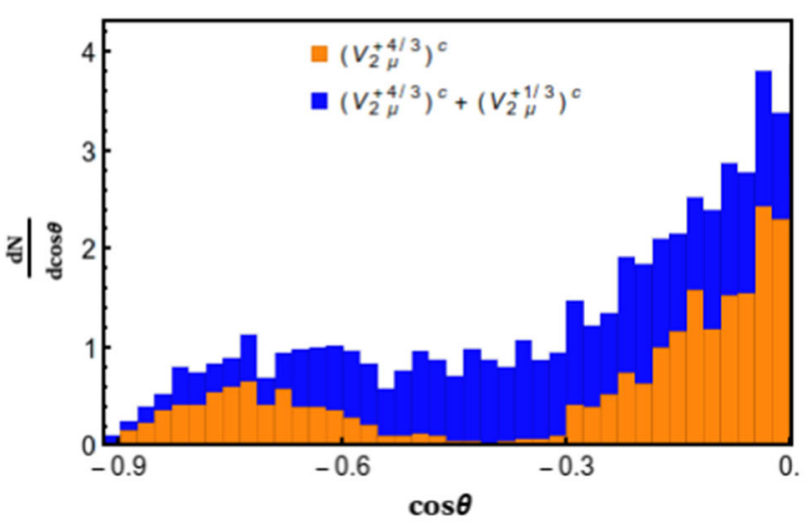

(c) Combined

Fig. 12 Angular distribution of photon with respect to the electron beam, at $\sqrt{s}=318.12 \mathrm{GeV}$ and $\mathcal{L}_{i n t}=100 \mathrm{fb}^{-1}$ for the associated production of $70 \mathrm{GeV}$ vector doublet leptoquark $V_{2 \mu}^{c}$ in CM frame.

The first plot shows the angular distribution of $\left(V_{2 \mu}^{+4 / 3}\right)^{c}$, the second one shows the same for $\left(V_{2 \mu}^{+1 / 3}\right)^{c}$, and the last one exhibits the angular distribution for signal and background together in the rest frame of interaction

The benchmark point (BP1) for the collider study along with the decay branching fractions and cross-sections with $p_{T}^{\gamma} \geq 20 \mathrm{GeV}$ are given by Tables 2,3 and 4 respectively. Like the scalar triplet case, here also we have two excitations

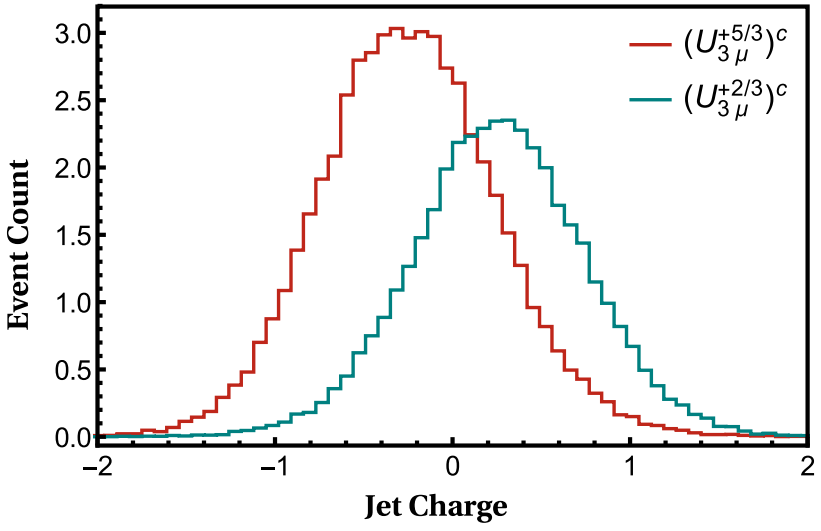

Fig. 13 Charge distribution (in the unit of $e$ ) of $c, s$-jets coming from the vector triplet leptoquark decays

Table 11 Number of events after the cumulative cuts for the vector triplet leptoquark $\boldsymbol{U}_{3 \mu}^{c}$ at $\sqrt{s}=318.12 \mathrm{GeV}$ and $\mathcal{L}_{i n t}=100 \mathrm{fb}^{-1}$. Significances with $\mathcal{L}_{\text {int }}=100 \mathrm{fb}^{-1}$ and $400 \mathrm{pb}^{-1}$ as well as integrated luminosity required for $5 \sigma$ significance at HERA are also estimated

\begin{tabular}{lll}
\hline Cuts & $\begin{array}{l}\text { Signal } \\
\left(U_{3 \mu}^{+5 / 3}\right)^{c}\end{array}$ & $\begin{array}{l}\text { SM } \\
+\left(U_{3 \mu}^{+2 / 3}\right)^{c}\end{array}$ \\
\hline $\mathcal{B}\left(\boldsymbol{U}_{3 \mu}^{c} \rightarrow \mu \bar{c} / \bar{s}\right): \mathrm{BP} 1$ & & \\
$\geqslant 1 \mu+1 j+1 \gamma$ & 69.4 & 52.1 \\
$\left|M_{l j}-M_{V_{2}}\right| \leq 10 \mathrm{GeV}$ & 53.6 & 39.6 \\
$1 \gamma_{p_{T}>20 \mathrm{GeV}}$ & & \\
$Q_{\text {Jet }}<-0.3$ & 24.9 & \\
$\sigma_{\text {Sig }}\left(\mathcal{L}_{\text {int }}=100 \mathrm{fb}^{-1}\right)$ & 4.6 & \\
$\sigma_{S i g}\left(\mathcal{L}_{\text {int }}=400 \mathrm{pb}^{-1}\right)$ & 0.28 & \\
$\mathcal{L}_{5 \sigma}\left(\right.$ in fb $\left.^{-1}\right)$ & 118.1 & \\
\hline
\end{tabular}

which can be produced i.e. $\left(U_{3 \mu}^{+5 / 3}\right)^{c}$ and $\left(U_{3 \mu}^{+2 / 3}\right)^{c}$. Similar to the previous cases here also we need to identify the charges of jets in order to distinguish $\left(U_{3 \mu}^{+5 / 3}\right)^{c}$ from $\left(U_{3 \mu}^{+2 / 3}\right)^{c}$. For this purpose the jet charge distributions are plotted in Fig. 13.

For the analysis of signal and background we again chose $\geqslant 1 \mu+1 j+1 \gamma$ final sate and reconstruct the leptoquark via the peak in invariant mass distribution of $\mu j$. We have implemented the first three cuts of Table 11 where $Q_{\text {Jet }}<$ -0.3 is instrumental in reducing the model contamination coming from $\left(U_{3 \mu}^{+2 / 3}\right)^{c}$.

Ready with the set up we plot the differential distribution with respect to the angle between the hard photon and the electron in CM frame, shown in Fig. 14. Figure 14a depicts the minimum of distribution for $\left(U_{3 \mu}^{+5 / 3}\right)^{c}$ about $\cos \theta=-\frac{1}{5}$, whereas Fig. 14b for $\left(U_{3 \mu}^{+2 / 3}\right)^{c}$ does not show any, as expected. We combine the distributions in Fig. 14c and the dip can still be observed.

In Table 11, we present the number of events after the cumulative cuts for $\left(U_{3 \mu}^{+5 / 3}\right)^{c}$ and SM plus $\left(U_{3 \mu}^{+2 / 3}\right)^{c}$. The sig- 


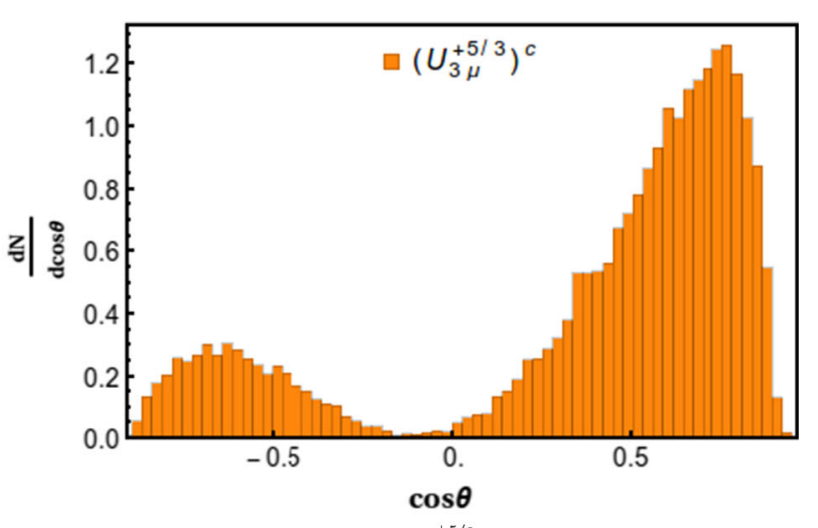

(a) $\left(U_{3 \mu}^{+5 / 3}\right)^{c}$

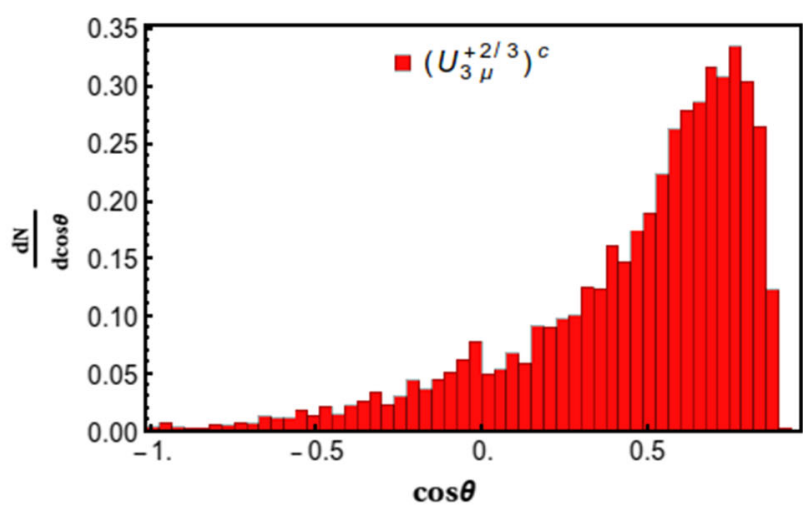

(b) $\left(U_{3 \mu}^{+2 / 3}\right)^{c}$

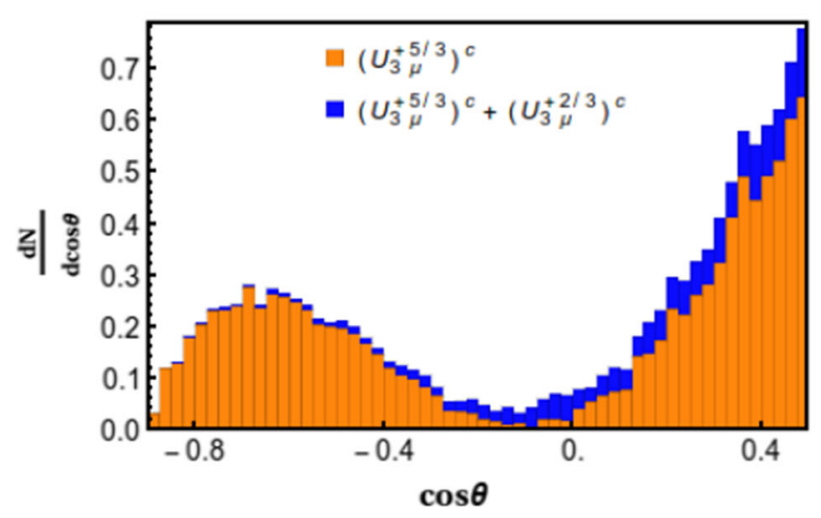

(c) Combined

Fig. 14 Angular distribution of photon with respect to the electron beam, at $\sqrt{s}=318.12 \mathrm{GeV}$ and $\mathcal{L}_{\text {int }}=100 \mathrm{fb}^{-1}$ for the associated production of $70 \mathrm{GeV}$ vector triplet leptoquark $\boldsymbol{U}_{3 \mu}^{c}$ in $\mathrm{CM}$ frame. The first plot shows the angular distribution of $\left(U_{3 \mu}^{+5 / 3}\right)^{c}$, the second one shows the same for $\left(U_{3 \mu}^{+2 / 3}\right)^{c}$, and the last one exhibits the angular distribution for signal and background together in the rest frame of interaction

nificance for $\left(U_{3 \mu}^{+5 / 3}\right)^{c}$ are calculated at $\sqrt{s}=318.12 \mathrm{GeV}$ : at $\mathcal{L}_{\text {int }}=100 \mathrm{fb}^{-1}, 400 \mathrm{pb}^{-1}$ at HERA. It is noticed that the signature of $\left(U_{3 \mu}^{+5 / 3}\right)^{c}$ can be determined over the model
Table 12 Beam and centre of mass energies along with integrated luminosities at $\mathrm{LHeC}$

\begin{tabular}{llll}
\hline$E_{p}$ & $E_{e}$ & $\sqrt{s}$ & $\mathcal{L}_{\text {int }}^{\text {projected }}$ \\
\hline $7 \mathrm{TeV}$ & $50 \mathrm{GeV}$ & $1183.2 \mathrm{GeV}$ & $2000 \mathrm{fb}^{-1}$ \\
\hline
\end{tabular}

contamination from $\left(U_{3 \mu}^{+2 / 3}\right)^{c}$ at an integrated luminosity of $\sim 118 \mathrm{fb}^{-1}$.

Clearly, as it follows from the signal-background table of events for all the leptoquark models exhibiting zeros in amplitude, with the size of the combined dataset of both the collaborations, H1 and ZEUS amounting to $400 \mathrm{pb}^{-1}$ for $e^{-} p$ collision, that it is not sufficient to observe a significant deviation for events exhibiting amplitude zeros. The highest significance obtained with this dataset is around $1.1 \sigma$ for the vector doublet leptoquark $V_{2 \mu}^{c}$ probe. However, for a futuristic collider operating with the same collision energy as HERA, if provide a dataset of size $100 \mathrm{fb}^{-1}$, the leptoquark signatures for $S_{3}^{c}$ and $V_{2 \mu}^{c}$ will definitely be measured with more than $5 \sigma$ significance through RAZ; the other two leptoquarks $R_{2}^{c}$ and $\boldsymbol{U}_{3 \mu}^{c}$ can also be observed with nearly $5 \sigma$ significance for the same integrated luminosity.

\section{Large hadron electron collider ( $\mathrm{LHeC})$}

The large hadron electron collider $(\mathrm{LHeC})$ is proposed at CERN, Geneva [91,92]. We present in Table 12 the technicalities for Run 6 of $\mathrm{LHeC}$, where a beam of $7 \mathrm{TeV}$ proton will be collided with a $50 \mathrm{GeV}$ electron beam giving rise to collisions at centre of momentum energy $\sim 1.2 \mathrm{TeV}$ with a projected luminosity of $2000 \mathrm{fb}^{-1}$.

For the collider simulation at $\mathrm{LHeC}$, we choose the mass of leptoquarks to be $900 \mathrm{GeV}$ with the couplings specified by BP2 scenario in Table 2. The corresponding branching fractions and production cross-sections associated with a photon $\left(p_{T}^{\gamma} \geq 20 \mathrm{GeV}\right)$ for different leptoquarks are gathered in Tables 3 and 4 respectively. It is important to mention that unlike HERA we had to select $|\eta|<4.5$ for all stable particles to confirm the occurrence of RAZ inside the detector since the lab frame of $\mathrm{LHeC}$ is highly boosted relative to the $\mathrm{CM}$ frame. As the position of RAZ in $e-p$ collider depends only on the charge of produced leptoquark and the production channels as well as the decay modes, taken for the simulation at $\mathrm{LHeC}$, are same with the preceding section we present here a comparative analysis of all the viable scalar and vector leptoquark models instead of presenting them in an elaborated repetitive discussion.

Similar to the earlier collider, we start with the kinematical distributions of scalar doublet leptoquark $R_{2}^{c}$ shown in Fig. 15. The sub-figures (a), (b) and (c) portray the distributions of $p_{T}$ for jet, muon and photon respectively. As anticipated, the 


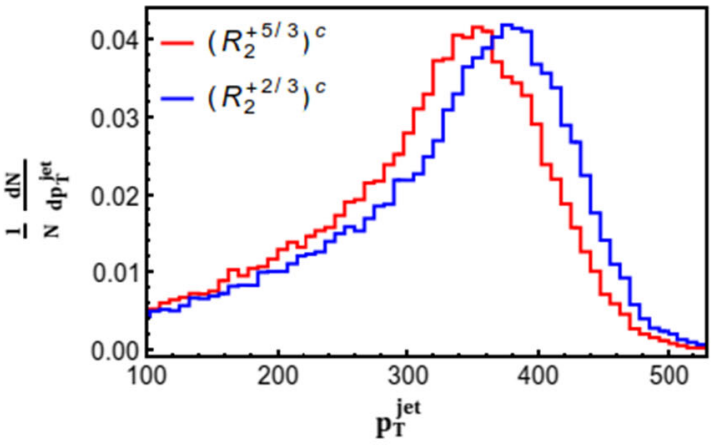

(a)

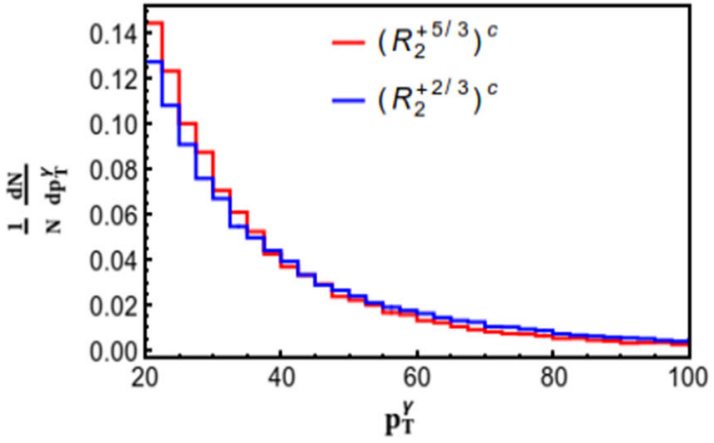

(c)

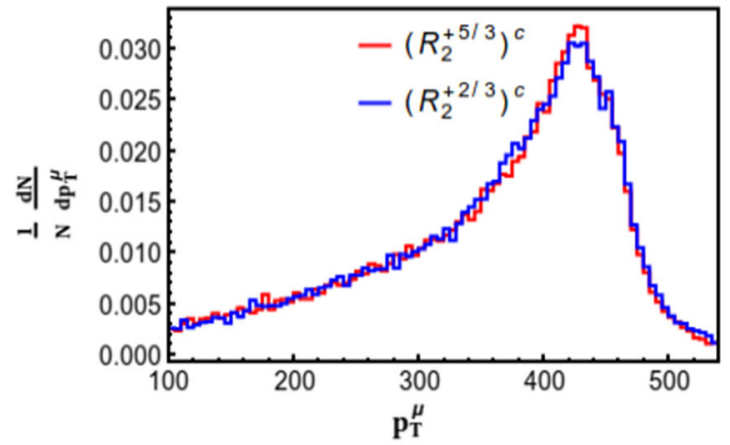

(b)

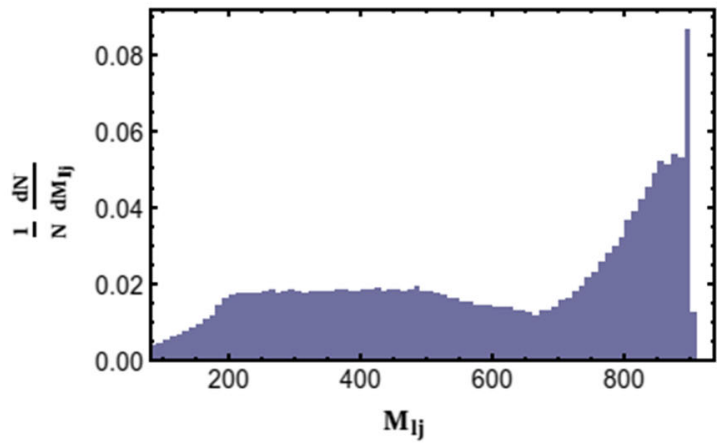

(d)

Fig. 15 Distributions of $p_{T}^{j}, p_{T}^{\mu}, p_{T}^{\gamma}$ and $M_{\ell j}$ at LHeC with $\sqrt{s}=1183.2 \mathrm{GeV}$ for the scalar-doublet leptoquark $R_{2}^{c}$ with mass $900 \mathrm{GeV}$

distributions of $p_{T}^{j}$ and $p_{T}^{\mu}$ show peaks roughly around half the mass of leptoquark. On the other hand, the sub-figure (d) displays the distribution for invariant mass of jet-muon pair which peaks at the leptoquark mass. However, in this case also the transverse momentum distributions for $\left(R_{2}^{+5 / 3}\right)^{c}$ and $\left(R_{2}^{+2 / 3}\right)^{c}$ approximately lie on top of each other and hence one should go for the jet-charge determination for the separation of the signatures of these two components, as described in the last section.

In this collider also, we probe the final state $\geqslant 1 \mu+$ $1 j+1 \gamma_{p_{T}>20 \mathrm{GeV}}$ with a reconstructed leptoquark satisfying $\left|M_{\ell j}-M_{\phi}\right| \leq 10 \mathrm{GeV}$. We show the angular distributions of photon with respect to its angle with electron beam in the CM frame for different leptoquark scenarios in Fig. 16. The first row portrays the angular distributions for all the scalar leptoquark scenarios whereas the second indicates the same for the vector ones. As has been seen for HERA, in case of doublet and triplet leptoquarks, the highest isospin component exhibits RAZ while the second excitation acts as model background showing no zero in the angular distribution. However, there still exits a dip in the combined plot of signal and background as can easily be observed from Figure 16.

Lastly, we collect the final state event numbers after the implication of different cuts for all the leptoquarks in Table 13 considering $\mathcal{L}_{i n t}=2000 \mathrm{fb}^{-1}$ and $\sqrt{s}=1183.2 \mathrm{GeV}$.
A significance of $5 \sigma$ is reachable for the vector doublet $V_{2 \mu}^{c}$ only, additionally, the scalar triplet $S_{3}^{c}$ can also be observed with more than $3 \sigma$ significance.

\section{FCC-he}

Finally we move to Future Circular Collider hadron-electron (FCC-he) [91,92] and probe the $\mathrm{TeV}$ scale leptoquark at higher energy as compared to $\mathrm{LHeC}$ in Sect. 6 where the cross-sections are relatively small (See Table 4). FCC-he is proposed in two phases, i.e. Phase I and Phase II. The FCCI will have the centre of momentum energy around $\sim 2.2$ $\mathrm{TeV}$, whereas FCC-II will have reach till $\sim 3.5 \mathrm{TeV}$ as can be seen from Table 14. The projected luminosity is around $2000 \mathrm{fb}^{-1}$.

\subsection{FCC I}

For FCC-I the leptoquark mass is chosen as $1.5 \mathrm{TeV}$ and like previous case, we present the kinematical distributions of scalar doublet leptoquark $R_{2}^{c}$ in Fig. 17. The sub-figures (a), (b), (c) describe the distribution of transverse momenta for jet, muon and photon respectively. It can be seen that jet and muon $p_{T}$ peak around half of the leptoquark mass i.e. $\sim 750 \mathrm{GeV}$ and certainly are very highly energetic. On the 


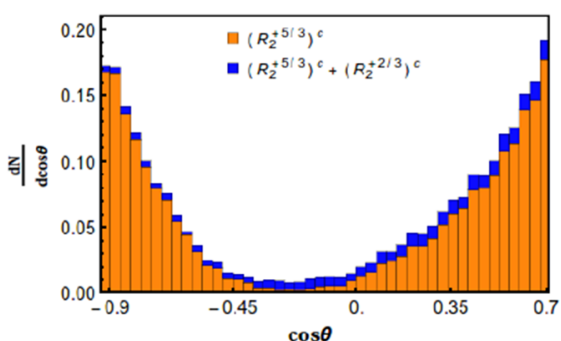

(a) $R_{2}^{c}$

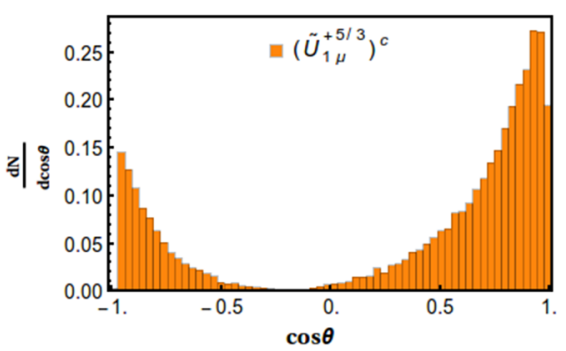

(d) $\widetilde{U}_{1 \mu}^{c}$

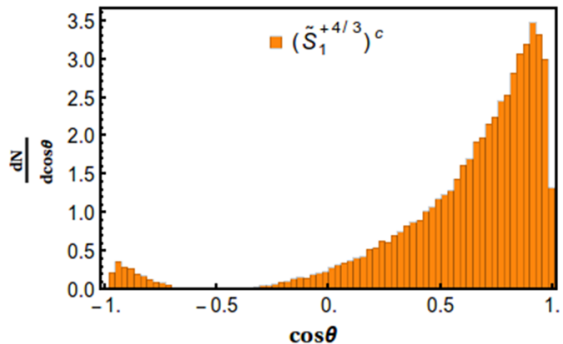

(b) $\widetilde{S}_{1}^{c}$

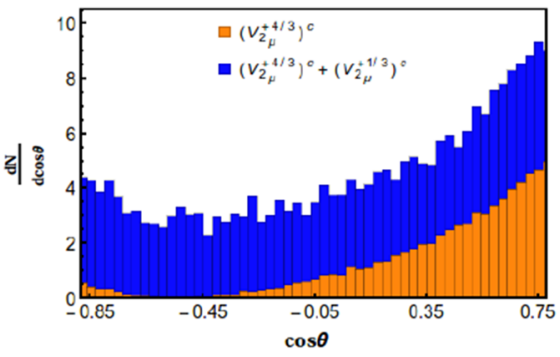

(e) $V_{2 \mu}^{c}$

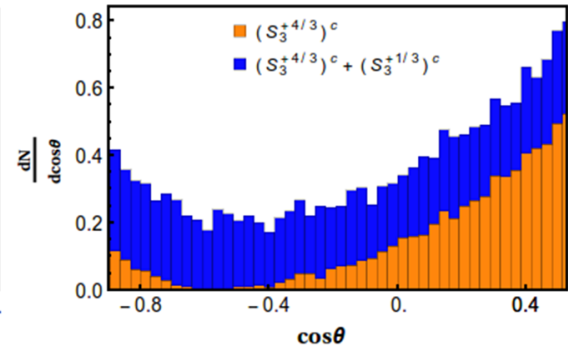

(c) $S_{3}^{c}$

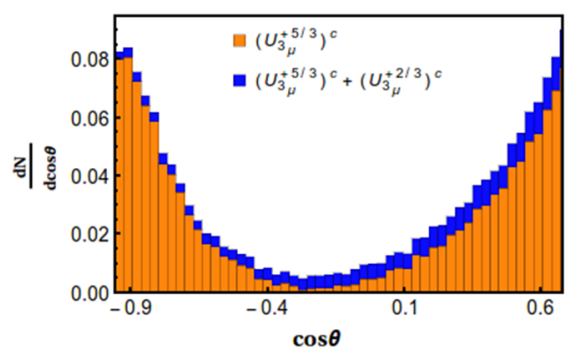

(f) $U_{3 \mu}^{c}$
Fig. 16 Distribution of cosine of the angle made by the photon with the electron beam, at $\sqrt{s}=1183.2 \mathrm{GeV}$ and $\mathcal{L}_{i n t}=2000 \mathrm{fb}^{-1}$. The sub-figures (a) $R_{2}^{c}$, (b) $\widetilde{S}_{1}^{c}$, (c) $\boldsymbol{S}_{3}^{c}$, (d) $\widetilde{U}_{1 \mu}^{c}$, (e) $V_{2 \mu}^{c}$ and (f) $\boldsymbol{U}_{3 \mu}^{c}$ show the angular distribution for associated production of the corresponding leptoquarks along with a photon at $\mathrm{LHeC}$

Table 13 Signal-background analysis for associated production of photon and different leptoquarks at $\mathrm{LHeC}$ with $\sqrt{s}=1183.2 \mathrm{GeV}$ and $\mathcal{L}_{\text {int }}=2000 \mathrm{fb}^{-1}$. The value of $Q_{\text {Jet }}^{\text {threshold }}$ has been chosen -0.3 for all the doublet and triplet leptoquarks

\begin{tabular}{|c|c|c|c|c|c|c|c|c|c|c|}
\hline \multirow[t]{3}{*}{ Cutss } & \multicolumn{5}{|c|}{ Scalar leptoquark } & \multicolumn{5}{|c|}{ Vector leptoquark } \\
\hline & \multirow{2}{*}{$\begin{array}{l}\text { Singlet } \\
\text { Signal } \\
\left(\widetilde{S}_{1}^{+4 / 3}\right)^{c}\end{array}$} & \multicolumn{2}{|l|}{ Doublet } & \multicolumn{2}{|l|}{ Triplet } & \multirow{2}{*}{$\begin{array}{l}\text { Singlet } \\
\text { Signal } \\
\left(\widetilde{U}_{1 \mu}^{+5 / 3}\right)^{c}\end{array}$} & \multicolumn{2}{|l|}{ Doublet } & \multicolumn{2}{|l|}{ Triplet } \\
\hline & & $\begin{array}{l}\text { Signal } \\
\left(R_{2}^{+5 / 3}\right)^{c}\end{array}$ & $\begin{array}{l}\mathrm{SM}+ \\
\left(R_{2}^{+2 / 3}\right)^{c}\end{array}$ & $\begin{array}{l}\text { Signal } \\
\left(S_{3}^{+4 / 3}\right)^{c}\end{array}$ & $\begin{array}{l}\mathrm{SM}+ \\
\left(S_{3}^{+1 / 3}\right)^{c}\end{array}$ & & $\begin{array}{l}\text { Signal } \\
\left(V_{2 \mu}^{+4 / 3}\right)^{c}\end{array}$ & $\begin{array}{l}\mathrm{SM}+ \\
\left(V_{2 \mu}^{+1 / 3}\right)^{c}\end{array}$ & $\begin{array}{l}\text { Signal } \\
\left(U_{3 \mu}^{+5 / 3}\right)^{c}\end{array}$ & $\begin{array}{l}\mathrm{SM}+ \\
\left(U_{3 \mu}^{+2 / 3}\right)^{c}\end{array}$ \\
\hline$\geqslant 1 \mu+1 j+1 \gamma$ & 76.3 & 22.7 & 8.5 & 79.3 & 215.8 & 7.9 & 454.2 & 3110.3 & 8.2 & 3.1 \\
\hline $\begin{array}{l}\left|M_{\ell j}-M_{\phi}\right| \leq 10 \mathrm{GeV} \\
+1 \gamma_{p_{T}>20 \mathrm{GeV}}\end{array}$ & 40.4 & 12.1 & 4.7 & 41.8 & 108.9 & 3.6 & 239.4 & 1568.1 & 4.1 & 1.6 \\
\hline$Q_{J e t}<Q_{J e t}^{\text {threshold }}$ & - & 5.1 & 0.53 & 18.8 & 14.8 & - & 108.1 & 217.1 & 2.8 & 0.46 \\
\hline$\sigma_{\text {Sig }}\left(\mathcal{L}_{i n t}=2000 \mathrm{fb}^{-1}\right)$ & - & 2.2 & & 3.2 & & - & 6.0 & & 1.5 & \\
\hline $\mathcal{L}_{5 \sigma}\left(\right.$ in $\left.\mathrm{fb}^{-1}\right)$ & - & 10300 & & 4900 & & - & 1400 & & 22200 & \\
\hline
\end{tabular}

other hand, sub-figure $(\mathrm{d})$ represent the invariant mass of the jet-muon pair and it peaks around the leptoquark mass $\sim 1.5$ $\mathrm{TeV}$.

The benchmark points for the collider study are described in Table 2 as BP3. The respective decay branching fractions of different leptoquark models are shown in Table 3 with the cross-sections for the leptoquarks production in association with a photon $\left(p_{T}^{\gamma} \geq 20 \mathrm{GeV}\right)$ given by Table 4 .

Like earlier scenarios, we look for the final state $(\geqslant$ $1 \mu+1 j+1 \gamma_{\left.p_{T}>20 \mathrm{GeV}\right)}$ with a reconstructed leptoquark ,i.e. $\left(\left|M_{\ell j}-M_{\phi}\right| \leq 10 \mathrm{GeV}\right)$, and plot the angular distribution with respect to the angle between the hard photon and electron in the CM frame, as illustrated in Fig. 18. Here the
Table 14 Beam and Centre of Mass energies along with projected integrated luminosities at FCC-he

\begin{tabular}{lllll}
\hline Stage & $E_{p}($ in TeV $)$ & $E_{e}($ in $\mathrm{GeV})$ & $\sqrt{s}($ in $\mathrm{GeV})$ & $\mathcal{L}_{\text {int }}^{\text {projected }}\left(\right.$ in fb $\left.^{-1}\right)$ \\
\hline I & 20 & 60 & 2190.2 & 2000 \\
II & 50 & 60 & 3464.1 & 2000 \\
\hline
\end{tabular}

upper panel shows the distributions from the scalar leptoquarks whereas lower panel describes the same for vector ones. Similar to HERA and LHeC, here also the zeros in their cross-sections are visible as minima in their angular dis- 


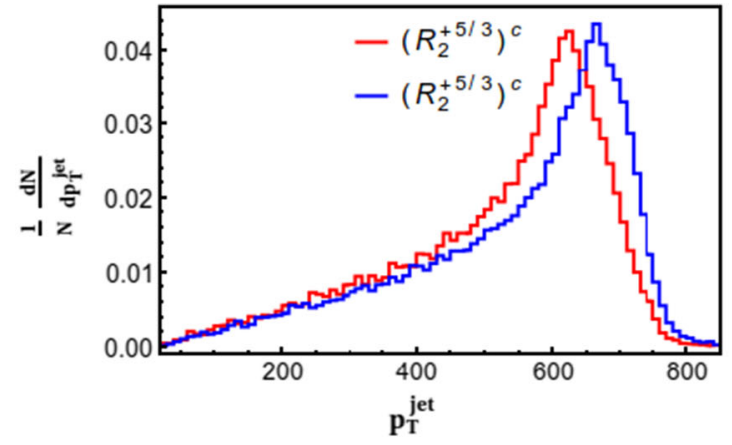

(a)

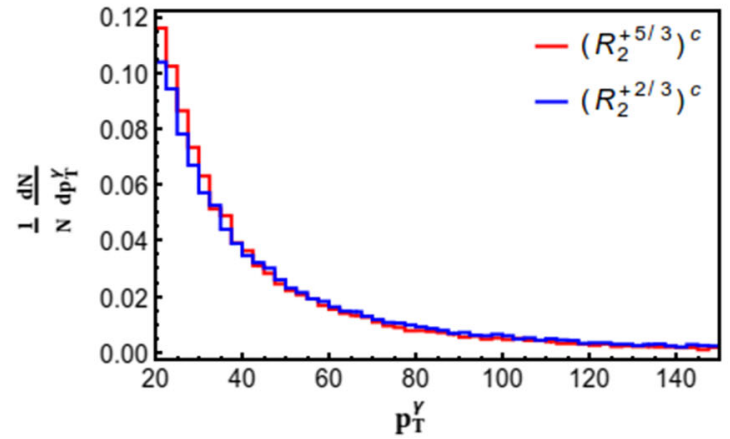

(c)

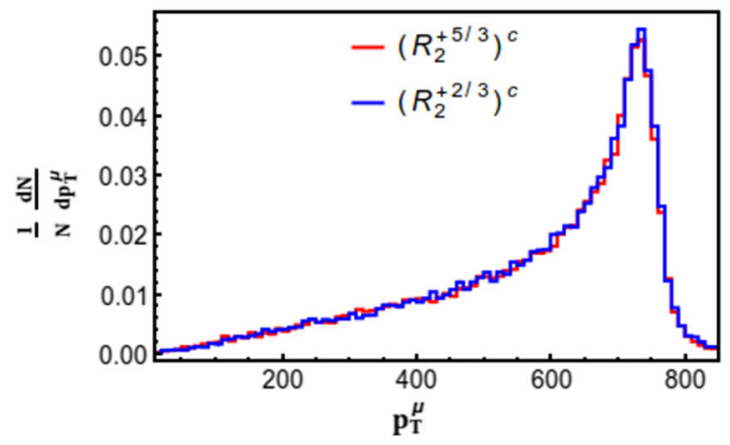

(b)

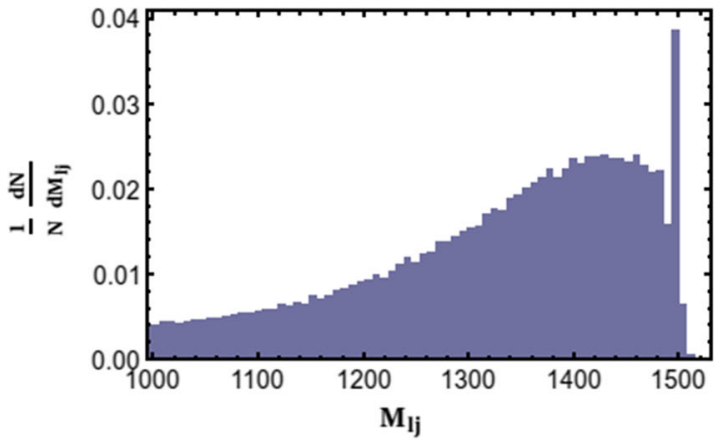

(d)

Fig. $17 p_{T}^{j}, p_{T}^{\ell}, p_{T}^{\gamma}$ and $M_{\ell j}$ distributions at FCC-I with $\sqrt{s}=2190.2 \mathrm{GeV}$ for the scalar-doublet leptoquark $R_{2}^{c}$ with mass $1.5 \mathrm{TeV}$

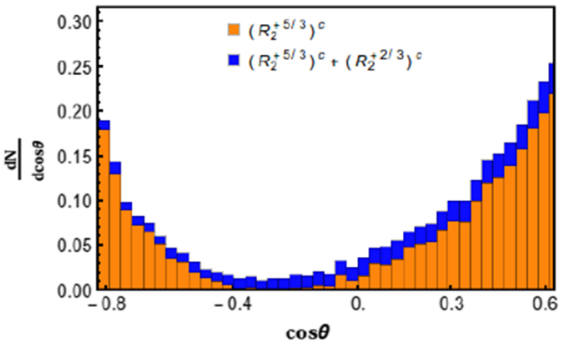

(a) $R_{2}^{c}$

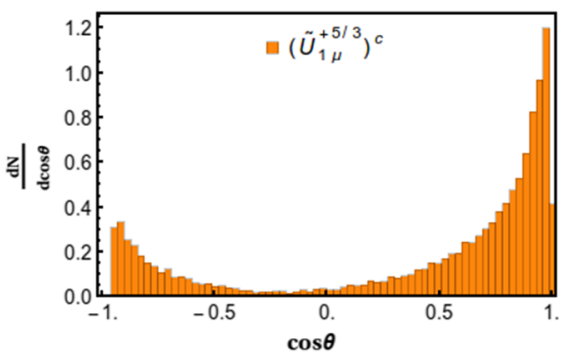

(d) $\widetilde{U}_{1 \mu}^{c}$

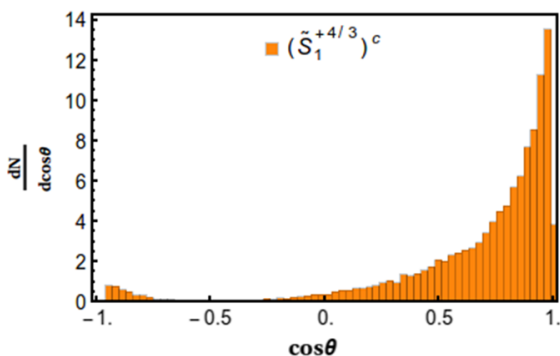

(b) $\widetilde{S}_{1}^{c}$

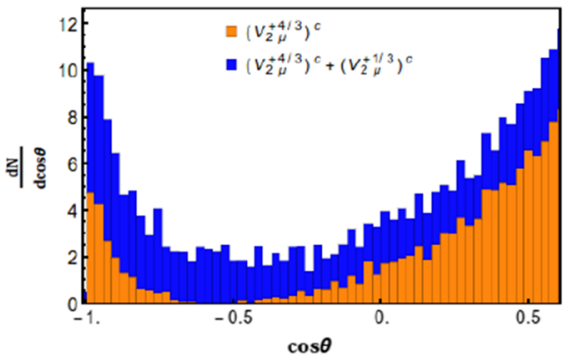

(e) $V_{2 \mu}^{c}$

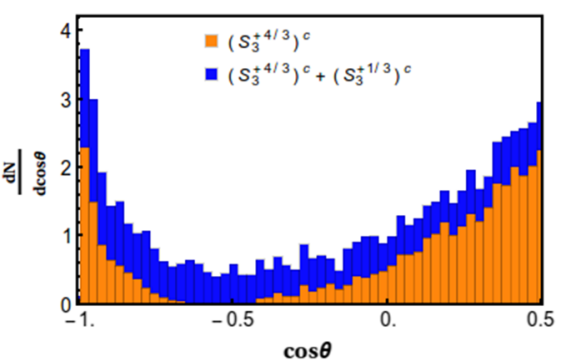

(c) $S_{3}^{c}$

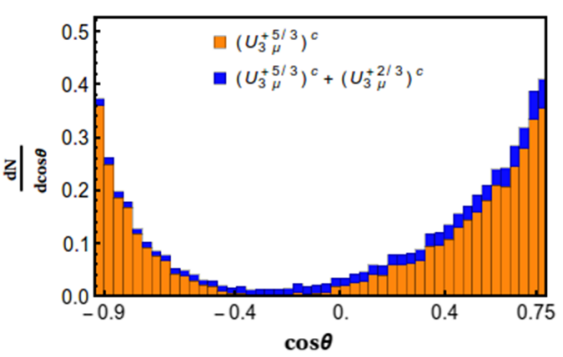

(f) $\boldsymbol{U}_{3 \mu}^{c}$

Fig. 18 Distribution of cosine of the angle made by the photon with the electron beam, at $\sqrt{s}=2190.2 \mathrm{GeV}$ and $\mathcal{L}_{\text {int }}=2000 \mathrm{fb}^{-1}$. The sub-figure a $R_{2}^{c}$, b $\widetilde{S}_{1}^{c}$, $\mathbf{S} S_{3}^{c}$, d $\widetilde{U}_{1 \mu}^{c}$, e $V_{2 \mu}^{c}$ and $\mathbf{f} U_{3 \mu}^{c}$ show the angular distribution for associated leptoquark production with a photon at FCC-I

tributions combined with SM plus the model backgrounds. Doublet and triplet leptoquarks which have more than one excitation get model contaminations from the other compo- nent of the multiplet that does not show any trace of zero, as explained before. Generally such different excitations have unalike decay modes leading to separate charged jets, i.e. up- 
Table 15 Signal-background analysis for associated production of photon and different leptoquarks at FCC I with $\sqrt{s}=2190.2 \mathrm{GeV}$ and $\mathcal{L}_{i n t}=2000 \mathrm{fb}^{-1}$. The value of $Q_{J e t}^{\text {threshold }}$ has been chosen 0 for $R_{2}^{c}$ and $\boldsymbol{U}_{3 \mu}^{c}$ while the same for $\boldsymbol{S}_{3}^{c}$ and $V_{2 \mu}^{c}$ is chosen to be -0.3

\begin{tabular}{|c|c|c|c|c|c|c|c|c|c|c|}
\hline \multirow[t]{3}{*}{ Cuts } & \multicolumn{5}{|c|}{ Scalar leptoquark } & \multicolumn{5}{|c|}{ Vector leptoquark } \\
\hline & \multirow{2}{*}{$\begin{array}{l}\text { Singlet } \\
\text { Signal } \\
\left(\widetilde{S}_{1}^{+4 / 3}\right)^{c}\end{array}$} & \multicolumn{2}{|l|}{ Doublet } & \multicolumn{2}{|l|}{ Triplet } & \multirow{2}{*}{$\begin{array}{l}\text { Singlet } \\
\text { Signal } \\
\left(\widetilde{U}_{1 \mu}^{+5 / 3}\right)^{c}\end{array}$} & \multicolumn{2}{|l|}{ Doublet } & \multicolumn{2}{|l|}{ Triplet } \\
\hline & & $\begin{array}{l}\text { Signal } \\
\left(R_{2}^{+5 / 3}\right)^{c}\end{array}$ & $\begin{array}{l}\mathrm{SM}+ \\
\left(R_{2}^{+2 / 3}\right)^{c}\end{array}$ & $\begin{array}{l}\text { Signal } \\
\left(S_{3}^{+4 / 3}\right)^{c}\end{array}$ & $\begin{array}{l}\mathrm{SM}+ \\
\left(S_{3}^{+1 / 3}\right)^{c}\end{array}$ & & $\begin{array}{l}\text { Signal } \\
\left(V_{2 \mu}^{+4 / 3}\right)^{c}\end{array}$ & $\begin{array}{l}\mathrm{SM}+ \\
\left(V_{2 \mu}^{+1 / 3}\right)^{c}\end{array}$ & $\begin{array}{l}\text { Signal } \\
\left(U_{3 \mu}^{+5 / 3}\right)^{c}\end{array}$ & $\begin{array}{l}\mathrm{SM}+ \\
\left(U_{3 \mu}^{+2 / 3}\right)^{c}\end{array}$ \\
\hline$\geqslant 1 \mu+1 j+1 \gamma$ & 569.8 & 42.4 & 15.2 & 600.9 & 1223.9 & 39.9 & 2397.5 & 4886.9 & 42.4 & 15.2 \\
\hline $\begin{array}{l}\left|M_{\ell j}-M_{\phi}\right| \leq 10 \mathrm{GeV} \\
+1 \gamma_{p_{T}>20 \mathrm{GeV}}\end{array}$ & 166.8 & 14.0 & 5.1 & 201.6 & 401.9 & 12.2 & 800.2 & 1621.4 & 14.0 & 5.1 \\
\hline$Q_{J e t}<Q_{J e t}^{\text {threshold }}$ & - & 9.7 & 1.4 & 91.2 & 52.5 & - & 364.4 & 209.3 & 9.7 & 1.5 \\
\hline$\sigma_{S i g}\left(\mathcal{L}_{i n t}=2000 \mathrm{fb}^{-1}\right)$ & - & 2.9 & & 7.6 & & - & 15.2 & & 2.9 & \\
\hline $\mathcal{L}_{5 \sigma}\left(\right.$ in $\left.\mathrm{fb}^{-1}\right)$ & - & 5900 & & 900 & & - & 200 & & 5900 & \\
\hline
\end{tabular}

or down-type, and thus can be distinguished by reconstructing the jet-charge.

We then gather the final state event numbers guided by the different cuts and present them in Table 15 at $2000 \mathrm{fb}^{-1}$ of integrated luminosity and $2190 \mathrm{GeV}$ of the centre of momentum energy. It is evident that a signal significance of $\sim 3 \sigma$ is achievable for most of the scenarios while more than $5 \sigma$ is for a few.

\subsection{FCC-II}

As FCC-II has elevated centre of momentum energy 3464 $\mathrm{GeV}$, we choose a relatively higher mass for the leptoquark i.e. $M_{\phi}=2 \mathrm{TeV}$ keeping all the couplings same as FCC-I. The benchmark points (BP4) for this scenario are already mentioned in Table 2 and the cross-sections for production of different leptoquarks in association with a photon $\left(p_{T}^{\gamma} \geq\right.$ $20 \mathrm{GeV}$ ) are enlisted in Table 4. The branching fractions for different leptoquarks under this scenario are almost same with those in case of FCC-I differing only at third decimal place and hence we do not show it explicitly in Table 3.

Due to heavier mass, the kinematical distributions for FCC-II will have tails at the higher momentum spectrum as depicted in Fig. 19. Figure 19a, b show the jet and muon $p_{T}$ distributions which have their peaks around half of the leptoquark mass i.e. $\sim 1 \mathrm{TeV}$, as during the on-shell production of leptoquarks the momenta are equally shared between the jets and the muons. Figure 19c, d describe the photon $p_{T}$ and invariant mass distributions of the jet-muon pair and the later peaks around the leptoquark mass of $\sim 2 \mathrm{TeV}$.

Similar to FCC-I, we present the angular distributions in CM frame of FCC-II for the different leptoquark scenarios with respect to the angle between the hard photon and the incoming electron in Fig. 20. The minima are visible, as expected, in different leptoquark scenarios, combined with SM plus model backgrounds, for the final state satisfying $\geqslant 1 \mu+1 j+1 \gamma_{p_{T}>20 \mathrm{GeV} \text { with the reconstructed leptoquark }}$ obeying $\left|M_{\ell j}-M_{\phi}\right| \leq 10 \mathrm{GeV}$. Later we present the final state numbers at FCC-II with centre of momentum energy of $3464.1 \mathrm{GeV}$ at an integrated luminosity of $2000 \mathrm{fb}^{-1}$ in Table 16. All the scenarios have signal significance over $5 \sigma$ in that given integrated luminosity.

\section{Discussion and conclusion}

Radiation amplitude zero is a well-established phenomenon in flavour Physics. It has mostly been used in determining the electromagnetic properties of $W$-boson. In this article, we use it to probe the signatures of the proposed particles leptoquarks in electron-proton collider. For our purpose, we explored the zeros in the angular distribution of the photon, produced in association with the leptoquark, in different electron proton colliders namely HERA, LHeC, FCC-I and FCC-II. In our simulation, we have worked with leptoquarks of different mass scales, viz. $70 \mathrm{GeV}$ to $2000 \mathrm{TeV}$, which are still allowed by various present and past collider bounds if proper couplings and branching fractions are chosen.

Scalar and vector singlet leptoquarks come with only one excitation thus there is no model contamination, which makes such typical angular distribution with zero at some kinematical point very easily detectable compared to the other scenarios. In case of doublets and triplets, the situation is little complicated. It is interesting to note that some of the components for these multiplets cannot be produced at any electronhandron collider due to the particular gauge structure of the interactions, whereas some of the other components, though get produced in $e-p$ collision, do not show RAZ within the physically allowed phase space. These second type of excitations perplex the circumstances for doublets and triplets since they tend to obscure the zeros in the distribution for the desired components. Such cases are handled by applying appropriate cuts which effectively reduce the model background. It is worth mentioning that since we have looked for 


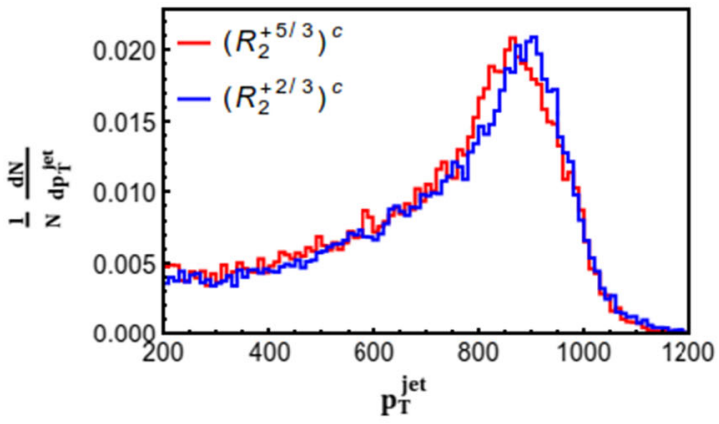

(a)

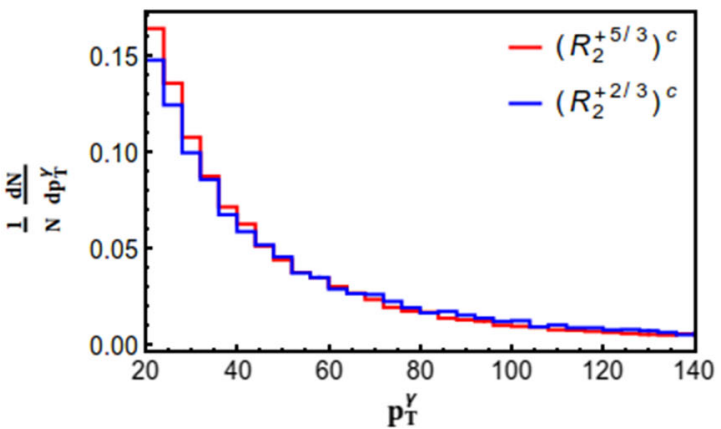

(c)

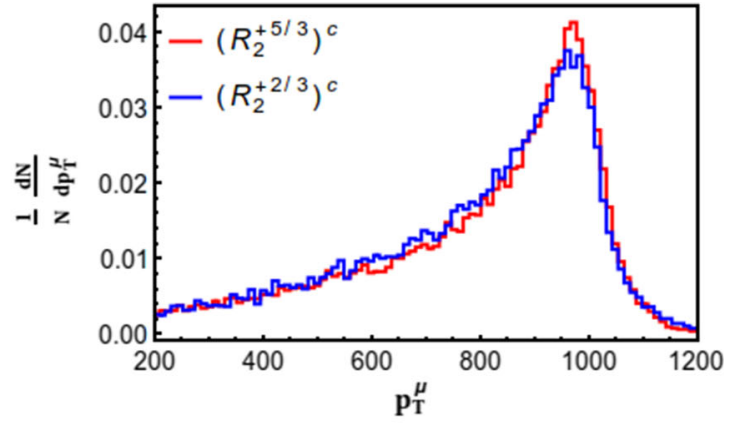

(b)

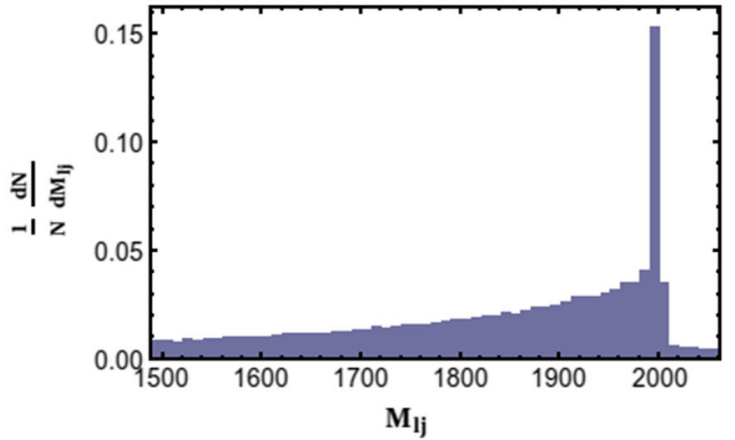

(d)

Fig. $19 p_{T}^{j}, p_{T}^{\ell}, p_{T}^{\gamma}$ and $M_{\ell j}$ distributions at FCC II with $\sqrt{s}=3464.1 \mathrm{GeV}$ for the scalar-doublet leptoquark $R_{2}^{c}$ with mass $2.0 \mathrm{TeV}$

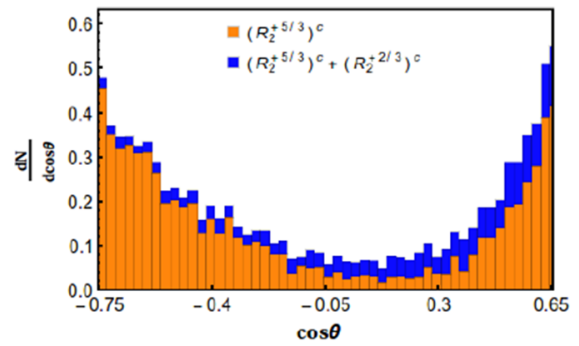

(a) $R_{2}^{c}$

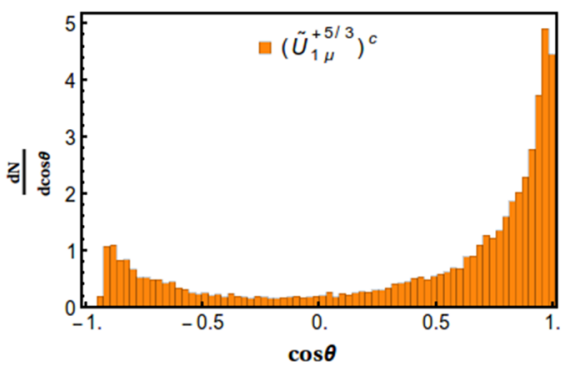

(d) $\tilde{U}_{1 \mu}^{c}$

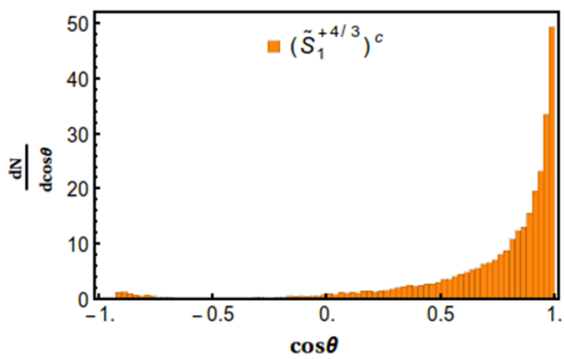

(b) $\widetilde{S}_{1}^{c}$

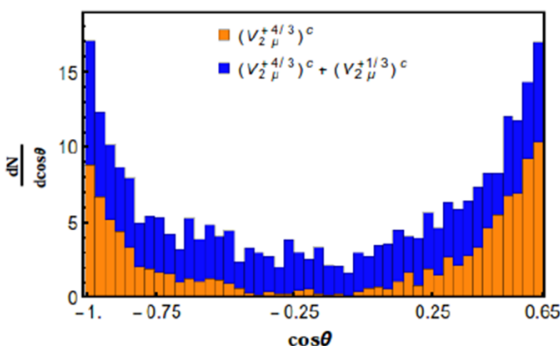

(e) $V_{2 \mu}^{c}$

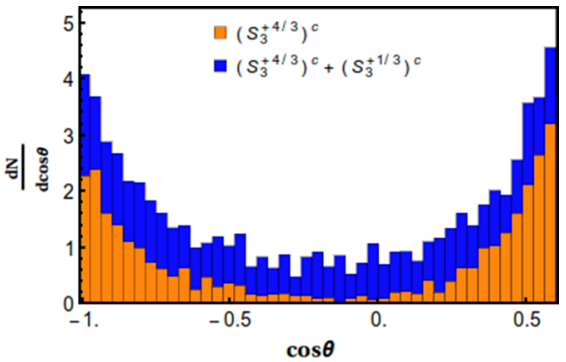

(c) $S_{3}^{c}$

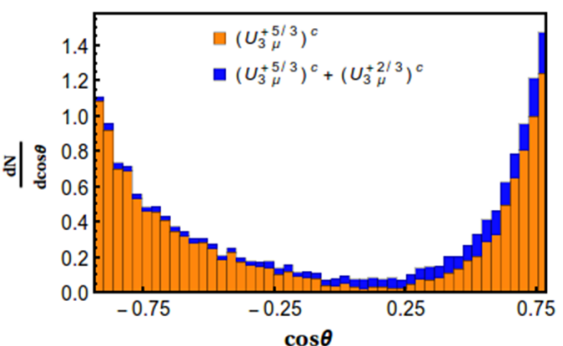

(f) $U_{3 \mu}^{c}$

Fig. 20 Distribution of cosine of the angle made by the photon with the electron beam, at $\sqrt{s}=3464.1 \mathrm{GeV}$ and $\mathcal{L}_{\text {int }}=2000 \mathrm{fb}^{-1}$. The sub-figure a $R_{2}^{c}$, b $\widetilde{S}_{1}^{c}, \mathbf{c} S_{3}^{c}$, d $\widetilde{U}_{1 \mu}^{c}$, e $V_{2 \mu}^{c}$ and $\mathbf{f} \boldsymbol{U}_{3 \mu}^{c}$ show the angular distribution for associated leptoquark production with a photon at FCC-II 
Table 16 Signal-background analysis for associated production of photon and different leptoquarks at FCC-II with $\sqrt{s}=3464.1 \mathrm{GeV}$ and $\mathcal{L}_{i n t}=2000 \mathrm{fb}^{-1}$. The value of $Q_{J e t}^{\text {threshold }}$ has been chosen 0 for $R_{2}^{c}$ and $\boldsymbol{U}_{3 \mu}^{c}$ while the same for $\boldsymbol{S}_{3}^{c}$ and $V_{2 \mu}^{c}$ is chosen to be -0.3

\begin{tabular}{|c|c|c|c|c|c|c|c|c|c|c|}
\hline \multirow[t]{3}{*}{ Cuts } & \multicolumn{5}{|c|}{ Scalar leptoquark } & \multicolumn{5}{|c|}{ Vector leptoquark } \\
\hline & \multirow{2}{*}{$\begin{array}{l}\text { Singlet } \\
\text { Signal } \\
\left(\widetilde{S}_{1}^{+4 / 3}\right)^{c}\end{array}$} & \multicolumn{2}{|l|}{ Doublet } & \multicolumn{2}{|l|}{ Triplet } & \multirow{2}{*}{$\begin{array}{l}\text { Singlet } \\
\text { Signal } \\
\left(\widetilde{U}_{1 \mu}^{+5 / 3}\right)^{c}\end{array}$} & \multicolumn{2}{|l|}{ Doublet } & \multicolumn{2}{|l|}{ Triplet } \\
\hline & & $\begin{array}{l}\text { Signal } \\
\left(R_{2}^{+5 / 3}\right)^{c}\end{array}$ & $\begin{array}{l}\mathrm{SM}+ \\
\left(R_{2}^{+2 / 3}\right)^{c}\end{array}$ & $\begin{array}{l}\text { Signal } \\
\left(S_{3}^{+4 / 3}\right)^{c}\end{array}$ & $\begin{array}{l}\mathrm{SM}+ \\
\left(S_{3}^{+1 / 3}\right)^{c}\end{array}$ & & $\begin{array}{l}\text { Signal } \\
\left(V_{2 \mu}^{+4 / 3}\right)^{c}\end{array}$ & $\begin{array}{l}\mathrm{SM}+ \\
\left(V_{2 \mu}^{+1 / 3}\right)^{c}\end{array}$ & $\begin{array}{l}\text { Signal } \\
\left(U_{3 \mu}^{+5 / 3}\right)^{c}\end{array}$ & $\begin{array}{l}\mathrm{SM}+ \\
\left(U_{3 \mu}^{+2 / 3}\right)^{c}\end{array}$ \\
\hline$\geqslant 1 \mu+1 j+1 \gamma$ & 1636.7 & 205.8 & 75.5 & 1781.6 & 2663.4 & 186.4 & 7051.4 & 10604.2 & 205.8 & 76.3 \\
\hline $\begin{array}{l}\left|M_{\ell j}-M_{\phi}\right| \leq 10 \mathrm{GeV} \\
+1 \gamma_{p_{T}>20 \mathrm{GeV}}\end{array}$ & 569.2 & 74.1 & 24.5 & 575.4 & 874.9 & 69.9 & 2322.6 & 3496.6 & 75.1 & 29.3 \\
\hline$Q_{J e t}<Q_{J e t}^{\text {threshold }}$ & - & 43.0 & 6.6 & 339.6 & 81.5 & - & 779.4 & 329.6 & 43.7 & 6.1 \\
\hline$\sigma_{\text {Sig }}\left(\mathcal{L}_{i n t}=2000 \mathrm{fb}^{-1}\right)$ & - & 6.1 & & 16.5 & & - & 23.4 & & 6.2 & \\
\hline $\mathcal{L}_{5 \sigma}\left(\right.$ in $\left.\mathrm{fb}^{-1}\right)$ & - & 1300 & & 200 & & - & 90 & & 1300 & \\
\hline
\end{tabular}

the muons in the final state as the charged lepton, the channel becomes SM background free absolutely.

The reconstruction of leptoquark via jet-muon invariant mass is crucial in order to establish the centre of momentum frame where we look for the zeros in angular distributions. The results at the centre of momentum energy of HERA are very promising and early data would give $5 \sigma$ signal significance for all the scenarios except scalar doublet and vector triplet ones which would take integrated luminosity of 100 $\mathrm{fb}^{-1}$.

On the other hand, the situation is a bit tight for LHeC. Due to various bounds from ATLAS and CMS, it is very difficult to have a leptoquark within the mass range 200-900 GeV. But centre of momentum energy available at $\mathrm{LHeC}$ is around 1.2 $\mathrm{TeV}$ and therefore, due to phase space suppression, it is quite challenging to achieve a good significance for the associated production of leptoquark with a photon.

In FCC-I, we have investigated the leptoquarks with mass 1.5 TeV where the cross-sections are not very encouraging and except scalar triplet and vector doublet scenarios a $5 \sigma$ reach is quite not possible with the integrated luminosity of $2000 \mathrm{fb}^{-1}$. The situation however changes when we upgrade to FCC-II and search for leptoquark with mass $2 \mathrm{TeV}$. In this collider $5 \sigma$ significance is achievable within the projected luminosity of $2000 \mathrm{fb}^{-1}$ for all the scenarios.

Finally, we find that the leptoquarks showing RAZ in $e$ $p$ collider do not exhibit zero in $e-\gamma$ collider while getting produced in association with a quark or anti-quark. Hence, in order to probe leptoquarks through the zeros of single photon tree level amplitude, it is necessary to study their production in both $e-p$ and $e-\gamma$ colliders.

Acknowledgements PB and AK acknowledge SERB CORE Grant CRG/2018/004971 and MATRICS Grant MTR/2020/000668 for the financial support towards the work.
Data Availability Statement This manuscript has no associated data or the data will not be deposited. [Authors' comment: The results in this paper are theoretical predictions related to the observation of RAZ during the production of proposed particles leptoquarks at e-p colliders. Our analysis is primarily based on the simulation by PYTHIA8. Hence, the results of this paper need to be confirmed by experiments in future. Any astrophysical or experimental data have not been analyzed in this paper.]

Open Access This article is licensed under a Creative Commons Attribution 4.0 International License, which permits use, sharing, adaptation, distribution and reproduction in any medium or format, as long as you give appropriate credit to the original author(s) and the source, provide a link to the Creative Commons licence, and indicate if changes were made. The images or other third party material in this article are included in the article's Creative Commons licence, unless indicated otherwise in a credit line to the material. If material is not included in the article's Creative Commons licence and your intended use is not permitted by statutory regulation or exceeds the permitted use, you will need to obtain permission directly from the copyright holder. To view a copy of this licence, visit http://creativecomm ons.org/licenses/by/4.0/.

Funded by $\mathrm{SCOAP}^{3}$.

\section{References}

1. K. Mikaelian, M. Samuel, D. Sahdev, The magnetic moment of weak bosons produced in $p p$ and $p \bar{p}$ collisions. Phys. Rev. Lett. 43, 746 (1979)

2. R. Brown, D. Sahdev, K. Mikaelian, $W^{ \pm} Z^{0}$ and $W^{ \pm} \gamma$ pair production in ve, $p p$, and $\bar{p} p$ collisions. Phys. Rev. D 20, 1164 (1979)

3. C. Goebel, F. Halzen, J. Leveille, Angular zeros of Brown, Mikaelian, Sahdev, and Samuel and the factorization of tree amplitudes in gauge theories. Phys. Rev. D 23, 2682-2685 (1981)

4. D.P. Zhu, Zeros in scattering amplitudes and the structure of nonabelian gauge theories. Phys. Rev. D 22, 2266 (1980)

5. N. Deshpande, X.-G. He, S. Oh, Amplitude zeros in radiative decays of scalar particles. Phys. Rev. D 51, 2295-2301 (1995). arXiv:hep-ph/9410373

6. S.J. Brodsky, R.W. Brown, Zeros in amplitudes: gauge theory and radiation interference. Phys. Rev. Lett. 49, 966 (1982) 
7. R.W. Brown, K. Kowalski, S.J. Brodsky, Classical radiation zeros in gauge theory amplitudes. Phys. Rev. D 28, 624 (1983). [Addendum: Phys. Rev. D 29, 2100-2104 (1984)]

8. G. Passarino, Physical null zones and radiation representation. Nucl. Phys. B 224, 265-288 (1983)

9. M. Samuel, Amplitude zeros. Phys. Rev. D 27, 2724-2731 (1983)

10. M. Samuel, A. Sen, G. Sylvester, M. Laursen, General criteria for radiation amplitude zeros. Phys. Rev. D 29, 994-999 (1984)

11. J. Stroughair, C. Bilchak, The determination of the $W$ anomalous magnetic moment in $p \bar{p} \rightarrow W \gamma X$.Z. Phys. C 26, 415-419(1984)

12. U. Baur, D. Zeppenfeld, Probing the $W W \gamma$ vertex at future hadron colliders. Nucl. Phys. B 308, 127-148 (1988)

13. J. Cortes, K. Hagiwara, F. Herzog, Testing the $W W \gamma$ coupling of the standard model at $p \bar{p}$ colliders. Nucl. Phys. B 278, 26 (1986)

14. G. Valenzuela, J. Smith, Experimental implications of finite width and $p_{T}$ effects in $W$ boson radiative production. Phys. Rev. D 31, $2787(1985)$

15. M.L. Laursen, M.A. Samuel, A. Sen, G. Tupper, Do amplitude zeros persist in higher order? Nucl. Phys. B 226, 429-436 (1983)

16. M. Laursen, M. Samuel, A. Sen, On the spoiling of amplitude (radiation) zeros at the one loop level and infrared finiteness. Phys. Rev. D 28, 650 (1983)

17. J. Smith, D. Thomas, W. van Neerven, QCD corrections to the reaction $p \bar{p} \rightarrow W \gamma X$. Z. Phys. C 44, 267 (1989)

18. J. Ohnemus, Order $\alpha_{s}$ calculations of hadronic $W^{ \pm} \gamma$ and $Z \gamma$ production. Phys. Rev. D 47, 940-955 (1993)

19. U. Baur, E.L. Berger, Probing the $W W \gamma$ vertex at the tevatron collider. Phys. Rev. D 41, 1476 (1990)

20. U. Baur, T. Han, J. Ohnemus, QCD corrections to hadronic $W \gamma$ production with nonstandard $W W \gamma$ couplings. Phys. Rev. D 48, 5140-5161 (1993). arXiv:hep-ph/9305314

21. U. Baur, S. Errede, G.L. Landsberg, Rapidity correlations in $W \gamma$ production at hadron colliders. Phys. Rev. D 50, 1917-1930 (1994). arXiv:hep-ph/9402282

22. R.M. Capdevilla, R. Harnik, A. Martin, The radiation valley and exotic resonances in $W \gamma$ production at the LHC. JHEP 03, 117 (2020). arXiv: 1912.08234

23. J.C. Pati, A. Salam, Unified lepton-hadron symmetry and a gauge theory of the basic interactions. Phys. Rev. D 8, 1240-1251 (1973)

24. J.C. Pati, A. Salam, Lepton number as the fourth color. Phys. Rev. D 10, 275-289 (1974). [Erratum: Phys. Rev. D 11, 703-703 (1975)]

25. H. Georgi, The state of the art—gauge theories. AIP Conf. Proc. 23, 575-582 (1975)

26. H. Georgi, S. Glashow, Unity of all elementary particle forces. Phys. Rev. Lett. 32, 438-441 (1974)

27. S. Dimopoulos, L. Susskind, Mass without scalars

28. E. Farhi, L. Susskind, Technicolor. Phys. Rep. 74, 277 (1981)

29. B. Schrempp, F. Schrempp, Light leptoquarks. Phys. Lett. B 153, 101-107 (1985)

30. J. Wudka, Composite leptoquarks. Phys. Lett. B 167, 337-342 (1986)

31. H.P. Nilles, Supersymmetry, supergravity and particle physics. Phys. Rep. 110, 1-162 (1984)

32. H.E. Haber, G.L. Kane, The search for supersymmetry: probing physics beyond the standard model. Phys. Rep. 117, 75-263 (1985)

33. J.L. Hewett, T.G. Rizzo, Much ado about leptoquarks: a comprehensive analysis. Phys. Rev. D 56, 5709-5724 (1997). arXiv:hep-ph/9703337

34. I. Doršner, S. Fajfer, A. Greljo, J. Kamenik, N. Košnik, Physics of leptoquarks in precision experiments and at particle colliders. Phys. Rep. 641, 1-68 (2016). arXiv:1603.04993

35. CELLO collaboration, H. Behrend et al., Search for light leptoquark bosons. Phys. Lett. B 178, 452-456 (1986). [Addendum: Phys. Lett. B 184, 417 (1987)]
36. JADE collaboration, W. Bartel et al., Search for leptoquarks and other new particles with lepton-hadron signature in $e^{+} e^{-}$interactions. Z. Phys. C 36, 15 (1987)

37. AMY collaboration, G. Kim et al., A search for leptoquark and colored lepton pair production in $e^{+} e^{-}$annihilations at TRISTAN. Phys. Lett. B 240, 243-249 (1990)

38. ALEPH collaboration, D. Decamp et al., Searches for new particles in $Z$ decays using the ALEPH detector. Phys. Rep. 216, 253-340 (1992)

39. L3 collaboration, O. Adriani et al., Results from the L3 experiment at LEP. Phys. Rep. 236, 1-146 (1993)

40. OPAL collaboration, G. Abbiendi et al., Search for pair produced leptoquarks in $e^{+} e^{-}$interactions at $\sqrt{s} \simeq 189 \mathrm{GeV}-209 \mathrm{GeV}$. Eur. Phys. J. C 31, 281-305 (2003). arXiv:hep-ex/0305053

41. DELPHI collaboration, P. Abreu et al., Search for Leptoquarks and FCNC in $e^{+} e^{-}$annihilations at $\sqrt{s}=183 \mathrm{GeV}$. Phys. Lett. B 446, 62-74 (1999). arXiv:hep-ex/9903072

42. H1 collaboration, F. Aaron et al., Search for lepton flavour violation at HERA. Phys. Lett. B 701, 20-30 (2011). arXiv:1103.4938

43. H1 collaboration, F. Aaron et al., Search for first generation leptoquarks in ep collisions at HERA. Phys. Lett. B 704, 388-396 (2011). arXiv: 1107.3716

44. ZEUS collaboration, H. Abramowicz et al., Search for firstgeneration leptoquarks at HERA. Phys. Rev. D 86, 012005 (2012). arXiv: 1205.5179

45. ZEUS collaboration, H. Abramowicz et al., Limits on contact interactions and leptoquarks at HERA. Phys. Rev. D 99092006 (2019). arXiv:1902.03048

46. UA2 collaboration, J. Alitti et al., A search for scalar leptoquarks at the CERN $\bar{p} p$ collider. Phys. Lett. B 274, 507-512 (1992)

47. CDF collaboration, D. Acosta et al., Search for first-generation scalar leptoquarks in $p \bar{p}$ collisions at $\sqrt{s}=1.96 \mathrm{TeV}$. Phys. Rev. D 72, 051107 (2005). arXiv:hep-ex/0506074

48. CDF collaboration, A. Abulencia et al., Search for secondgeneration scalar leptoquarks in $p \bar{p}$ collisions at $\sqrt{s}=1.96 \mathrm{TeV}$. Phys. Rev. D 73, 051102 (2006). arXiv:hep-ex/0512055

49. CDF collaboration, T. Aaltonen et al., Search for third generation vector leptoquarks in $p \bar{p}$ collisions at $\sqrt{s}=1.96 \mathrm{TeV}$. Phys. Rev. D 77, 091105 (2008). arXiv:0706.2832

50. D0 collaboration, V. Abazov et al., Search for pair production of second generation scalar leptoquarks. Phys. Lett. B 671, 224-232 (2009). arXiv:0808.4023

51. D0 collaboration, V. M. Abazov et al., Search for scalar bottom quarks and third-generation leptoquarks in $p \bar{p}$ collisions at $\sqrt{s}=$ 1.96 TeV. Phys. Lett. B 693, 95-101 (2010). arXiv:1005.2222

52. D0 collaboration, V.M. Abazov et al., Search for first generation leptoquark pair production in the electron + missing energy + jets final state. Phys. Rev. D 84, 071104 (2011). arXiv:1107.1849

53. ATLAS collaboration, M. Aaboud et al., Searches for thirdgeneration scalar leptoquarks in $\sqrt{s}=13 \mathrm{TeV}$ pp collisions with the ATLAS detector. JHEP 06, 144 (2019). arXiv:1902.08103

54. ATLAS collaboration, M. Aaboud et al., Searches for scalar leptoquarks and differential cross-section measurements in dileptondijet events in proton-proton collisions at a centre-of-mass energy of $\sqrt{s}=13 \mathrm{TeV}$ with the ATLAS experiment. Eur. Phys. J. C 79, 733 (2019). arXiv: 1902.00377

55. CMS collaboration, A. M. Sirunyan et al., Search for pair production of first-generation scalar leptoquarks at $\sqrt{s}=13$ TeV. Phys. Rev. D 99, 052002 (2019). arXiv: 1811.01197

56. CMS collaboration, A.M. Sirunyan et al., Constraints on models of scalar and vector leptoquarks decaying to a quark and a neutrino at $\sqrt{s}=13$ TeV. Phys. Rev. D 98, 032005 (2018). arXiv: 1805.10228

57. CMS collaboration, A.M. Sirunyan et al., Search for thirdgeneration scalar leptoquarks decaying to a top quark and a 
$\tau$ lepton at $\sqrt{s}=13$ TeV. Eur. Phys. J. C 78, 707 (2018). arXiv: 1803.02864

58. CMS collaboration, A.M. Sirunyan et al., Search for pair production of second-generation leptoquarks at $\sqrt{s}=13$ TeV. Phys. Rev. D 99, 032014 (2019). arXiv: 1808.05082

59. CMS collaboration, A.M. Sirunyan et al., Search for heavy neutrinos and third-generation leptoquarks in hadronic states of two $\tau$ leptons and two jets in proton-proton collisions at $\sqrt{s}=13 \mathrm{TeV}$. JHEP 03, 170 (2019). arXiv:1811.00806

60. W. Buchmuller, D. Wyler, Constraints on SU(5) type leptoquarks. Phys. Lett. B 177, 377-382 (1986)

61. W. Buchmuller, R. Ruckl, D. Wyler, Leptoquarks in lepton-quark collisions. Phys. Lett. B 191, 442-448 (1987). [Erratum: Phys. Lett. B 448, 320-320 (1999)]

62. J. Hewett, S. Pakvasa, Leptoquark production in hadron colliders. Phys. Rev. D 37, 3165 (1988)

63. A. Belyaev, C. Leroy, R. Mehdiyev, A. Pukhov, Leptoquark single and pair production at LHC with CalcHEP/CompHEP in the complete model. JHEP 09, 005 (2005). arXiv:hep-ph/0502067

64. P. Bandyopadhyay, R. Mandal, Revisiting scalar leptoquark at the LHC. Eur. Phys. J. C 78, 491 (2018). arXiv:1801.04253

65. A. Bhaskar, D. Das, B. De, S. Mitra, Enhancing scalar productions with leptoquarks at the LHC. Phys. Rev. D 102, 035002 (2020). arXiv:2002.12571

66. K. Chandak, T. Mandal, S. Mitra, Hunting for scalar leptoquarks with boosted tops and light leptons. Phys. Rev. D 100, 075019 (2019). arXiv: 1907.11194

67. T. Mandal, S. Mitra, S. Seth, Single productions of colored particles at the lhc: an example with scalar leptoquarks. JHEP 07, 028 (2015). arXiv: 1503.04689

68. A. Alves, O.J.T. Eboli, G. Grilli Di Cortona, R.R. Moreira, Indirect and monojet constraints on scalar leptoquarks. Phys. Rev. D 99, 095005 (2019). arXiv: 1812.08632

69. I. Doršner, S. Fajfer, M. Patra, A comparative study of the $S_{1}$ and $U_{1}$ leptoquark effects in the light quark regime. Eur. Phys. J. C 80, 204 (2020). arXiv: 1906.05660

70. S. Mandal, M. Mitra, N. Sinha, Probing leptoquarks and heavy neutrinos at the LHeC. Phys. Rev. D 98, 095004 (2018). arXiv: 1807.06455

71. R. Padhan, S. Mandal, M. Mitra, N. Sinha, Signatures of $\tilde{R}_{2}$ class of Leptoquarks at the upcoming ep colliders. Phys. Rev. D 101, 075037 (2020). arXiv: 1912.07236

72. J. Fuentes-Martín, G. Isidori, M. König, N. Selimović, Vector leptoquarks beyond tree level. Phys. Rev. D 101, 035024 (2020). arXiv: 1910.13474

73. M.J. Baker, J. Fuentes-Martín, G. Isidori, M. König, High- $p_{T}$ signatures in vector-leptoquark models. Eur. Phys. J. C 79, 334 (2019). arXiv: 1901.10480

74. G. Bhattacharyya, J.R. Ellis, K. Sridhar, Bounds on the masses and couplings of leptoquarks from leptonic partial widths of the Z. Phys. Lett. B 336, 100-106 (1994). [Erratum: Phys. Lett. B 338, 522-523 (1994)]. arXiv:hep-ph/9406354

75. J. Hewett, T. Rizzo, Leptoquark signals at $e^{+} e^{-}$colliders. Phys. Rev. D 36, 3367 (1987)

76. T. Plehn, H. Spiesberger, M. Spira, P. Zerwas, Formation and decay of scalar leptoquarks/squarks in ep collisions. Z. Phys. C 74, 611-614 (1997). arXiv:hep-ph/9703433

77. M. Kramer, T. Plehn, M. Spira, P. Zerwas, Pair production of scalar leptoquarks at the Tevatron. Phys. Rev. Lett. 79, 341-344 (1997). arXiv:hep-ph/9704322

78. F. Cuypers, Leptoquark production in $e^{-} \gamma$ scattering. Nucl. Phys. B 474, 57-71 (1996). arXiv:hep-ph/9508397

79. O.J. Eboli, E. Gregores, M. Magro, P. Mercadante, S. Novaes, Searching for leptoquarks in electron-photon collisions. Phys. Lett. B 311, 147-152 (1993). arXiv:hep-ph/9306229
80. H. Nadeau, D. London, Leptoquarks at e gamma colliders. Phys. Rev. D 47, 3742-3749 (1993). arXiv:hep-ph/9303238

81. S. Atag, O. Cakir, Pair production of scalar leptoquarks at $\mathrm{TeV}$ energy gamma p colliders. Phys. Rev. D 49, 5769-5772 (1994)

82. S. Atag, A. Celikel, S. Sultansoy, Scalar leptoquark production at TeV energy gamma p colliders. Phys. Lett. B 326, 185-189 (1994)

83. A. Dobado, M. Herrero, C. Munoz, Production of leptoquarks from superstring models in $e p$ colliders. Phys. Lett. B 191, 449455 (1987)

84. V. Ilyin, A. Pukhov, V. Savrin, A. Semenov, W. von Schlippe, Single leptoquark production associated with hard photon emission in ep collisions at high-energies. Phys. Lett. B 351, 504509 (1995). [Erratum: Phys. Lett. B 352, 500-500 (1995)]. arXiv:hep-ph/9503401

85. J.F. Gunion, E. Ma, Production of $E(6)$ scalar leptoquarks in $e p$ collisions. Phys. Lett. B 195, 257-264 (1987)

86. P. Bandyopadhyay, S. Dutta, A. Karan, Investigating the production of leptoquarks by means of zeros of amplitude at photon electron collider. Eur. Phys. J. C 80, 573 (2020). arXiv:2003.11751

87. P. Bandyopadhyay, S. Dutta, M. Jakkapu, Exploring angular distributions at the LHC. arXiv:2007.12997

88. A. Angelescu, D. Bečirević, D. Faroughy, O. Sumensari, Closing the window on single leptoquark solutions to the $B$-physics anomalies. JHEP 10, 183 (2018). arXiv: 1808.08179

89. HERA-A Proposal for a Large Electron Proton Colliding Beam Facility at DESY (1981)

90. M. Klein, R. Yoshida, Collider Physics at HERA. Prog. Part. Nucl. Phys. 61, 343-393 (2008). arXiv:0805.3334

91. LHeC, FCC-he Study Group collaboration, P. Agostini et al., The large hadron-electron collider at the HL-LHC. arXiv:2007.14491

92. F. Bordry, M. Benedikt, O. Brüning, J. Jowett, L. Rossi, D. Schulte et al., Machine parameters and projected luminosity performance of proposed future colliders at CERN. arXiv:1810.13022

93. S. Davidson, D.C. Bailey, B.A. Campbell, Model independent constraints on leptoquarks from rare processes. Z. Phys. C 61, 613-644 (1994). arXiv:hep-ph/9309310

94. A.J. Davies, X.-G. He, Tree level scalar fermion interactions consistent with the symmetries of the standard model. Phys. Rev. D 43, 225-235 (1991)

95. Particle Data Group, P.A. Zyla et al., Review of particle physics. Prog. Theor. Exp. Phys. 2020, 083C01 (2020)

96. M. Leurer, A Comprehensive study of leptoquark bounds. Phys. Rev. D 49, 333-342 (1994). arXiv:hep-ph/9309266

97. M. Leurer, Bounds on vector leptoquarks. Phys. Rev. D 50, 536541 (1994). arXiv:hep-ph/9312341

98. M. Carpentier, S. Davidson, Constraints on two-lepton, two quark operators. Eur. Phys. J. C 70, 1071-1090 (2010). arXiv:1008.0280

99. R. Mandal, A. Pich, Constraints on scalar leptoquarks from lepton and kaon physics. JHEP 12, 089 (2019). arXiv:1908.11155

100. H1 collaboration, C. Adloff et al., Observation of events at very high $Q^{2}$ in $e p$ collisions at HERA. Z. Phys. C 74, 191-206 (1997). arXiv:hep-ex/9702012

101. ZEUS collaboration, J. Breitweg et al., Comparison of ZEUS data with standard model predictions for $e^{+} p \rightarrow e^{+} X$ scattering at high $\mathrm{x}$ and $Q^{2}$. Z. Phys. C 74, 207-220 (1997). arXiv:hep-ex/9702015

102. J. Blumlein, E. Boos, A. Kryukov, Leptoquark pair production in hadronic interactions. Z. Phys. C 76, 137-153 (1997). arXiv:hep-ph/9610408

103. ZEUS collaboration, S. Chekanov et al., A Search for resonance decays to lepton + jet at HERA and limits on leptoquarks. Phys. Rev. D 68, 052004 (2003). arXiv:hep-ex/0304008

104. CDF collaboration, F. Abe et al., A search for second generation leptoquarks in $p \bar{p}$ collisions at $\sqrt{s}=1.8 \mathrm{TeV}$. Phys. Rev. Lett. 75, 1012-1016 (1995) 
105. CDF collaboration, F. Abe et al., Search for third generation leptoquarks in $\bar{p} p$ collisions at $\sqrt{s}=1.8 \mathrm{TeV}$. Phys. Rev. Lett. 78, 2906-2911 (1997)

106. F. Staub, SARAH 4: A tool for (not only SUSY) model builders. Comput. Phys. Commun. 185, 1773-1790 (2014). arXiv: 1309.7223

107. A. Belyaev, N.D. Christensen, A. Pukhov, CalcHEP 3.4 for collider physics within and beyond the Standard Model. Comput. Phys. Commun. 184, 1729-1769 (2013). arXiv:1207.6082

108. NNPDF collaboration, E.R. Nocera, R.D. Ball, S. Forte, G. Ridolfi, J. Rojo, A first unbiased global determination of polarized PDFs and their uncertainties. Nucl. Phys. B 887, 276-308 (2014). arXiv:1406.5539

109. T. Sjöstrand, S. Mrenna, P.Z. Skands, A brief introduction to PYTHIA 8.1. Comput. Phys. Commun. 178, 852-867 (2008). arXiv:0710.3820

110. T. Sjöstrand, S. Ask, J.R. Christiansen, R. Corke, N. Desai, P. Ilten et al., An introduction to PYTHIA 8.2. Comput. Phys. Commun. 191, 159-177 (2015). arXiv:1410.3012
111. M. Cacciari, G.P. Salam, G. Soyez, FastJet user manual. Eur. Phys. J. C 72, 1896 (2012). arXiv:1111.6097

112. D. Krohn, M.D. Schwartz, T. Lin, W.J. Waalewijn, Jet charge at the LHC. Phys. Rev. Lett. 110, 212001 (2013). arXiv:1209.2421

113. ATLAS, CMS collaboration, S. Tokar, Jet charge determination at the LHC, in Parton radiation and fragmentation from $\mathrm{LHC}$ to FCC-ee, pp. 79-84, 2 (2017)

114. CMS collaboration, A.M. Sirunyan et al., Measurements of jet charge with dijet events in pp collisions at $\sqrt{s}=8 \mathrm{TeV}$. JHEP 10, 131 (2017). arXiv: 1706.05868 